\title{
Star-forming Environments throughout the M101 Group
}

\author{
Aaron E. Watkins ${ }^{1,2}$, J. Christopher Mihos $^{1}$ (D), and Paul Harding ${ }^{1}$ (D) \\ ${ }^{1}$ Department of Astronomy, Case Western Reserve University, Cleveland, OH 44106, USA \\ ${ }^{2}$ Astronomy Research Unit, University of Oulu, FI-90014, Finland \\ Received 2017 August 3; revised 2017 September 19; accepted 2017 September 26; published 2017 December 11
}

\begin{abstract}
We present a multiwavelength study of star formation within the nearby M101 Group, including new deep $\mathrm{H} \alpha$ imaging of M101 and its two companions. We perform a statistical analysis of the $\mathrm{H} \alpha$-to-FUV flux ratios in $\mathrm{H}$ II regions located in three different environments: M101's inner disk, M101's outer disk, and M101's lowermass companion galaxy NGC 5474. We find that, once bulk radial trends in extinction are taken into account, both the median and scatter in $F_{\mathrm{H} \alpha} / F_{\mathrm{FUV}}$ in $\mathrm{H}$ II regions are invariant across all of these environments. Also, using Starburst 99 models, we are able to qualitatively reproduce the distributions of $F_{\mathrm{H} \alpha} / F_{\mathrm{FUV}}$ throughout these different environments using a standard Kroupa initial mass function (IMF); hence, we find no need to invoke truncations in the upper-mass end of the IMF to explain the young star-forming regions in the M101 Group even at extremely low surface density. This implies that star formation in low-density environments differs from star formation in highdensity environments only by intensity and not by cloud-to-cloud physics.
\end{abstract}

Key words: galaxies: evolution - galaxies: individual (M101, NGC 5474) - galaxies: spiral - galaxies: star formation

\section{Introduction}

The extended, low surface brightness (LSB) outer disks of galaxies are a poor fit to idealized models of galaxy formation theory. Absent extenuating circumstances, $\Lambda \mathrm{CDM}$ predicts that galaxies form "inside-out," hence are youngest at their largest radii. Yet real galaxies' often smooth, red outer isophotes imply the opposite (e.g., Bakos et al. 2008; Zheng et al. 2015; Laine et al. 2016). In fact, old red giant branch (RGB) stars typically have longer scale lengths than main-sequence stars (e.g., Davidge 2003; Vlajić et al. 2009, 2011), and any young stars present in outer disks tend to be sparsely distributed (e.g., Barker et al. 2007; Davidge 2010). Outer disks are not simply an LSB continuation of inner disks.

Star formation is also inefficient in outer disks, with gas consumption timescales exceeding a Hubble time (Thilker et al. 2007; Bigiel et al. 2010). This is similar to LSB galaxies (e.g., McGaugh \& Bothun 1994; Burkholder et al. 2001; Boissier et al. 2008), suggesting that star formation physics changes in low-density environments. Jeans stability criteria suggest that low gas column density results in depressed or truncated star formation (with an apparent threshold below around $\Sigma_{\mathrm{H}_{\mathrm{I}}} \sim 10^{20}-10^{21} \mathrm{~cm}^{-2}$; e.g., Hunter \& Gallagher 1986; Skillman 1987; van der Hulst et al. 1987), but star formation may also be suppressed on large scales via dynamically induced stability (e.g., Zasov \& Simakov 1988; Kennicutt 1989). The latter suggests that disks should have a star formation truncation radius (Martin \& Kennicutt 2001), with star formation taking place beyond this only in local highdensity pockets (e.g., Courtes \& Cruvellier 1961; Ferguson et al. 1998; Gil de Paz et al. 2005; Thilker et al. 2005).

Despite its scarcity and inefficiency, this in situ outer disk star formation could fully account for all of the outer disk stellar mass in some galaxies (depending on the star formation history (SFH); Zaritsky \& Christlein 2007). However, outer disk star formation is present in only $\sim 4 \%-14 \%$ of starforming galaxies out to $z=0.05$ (Lemonias et al. 2011); hence, it may not be sufficient to explain outer disk formation in general. It also may not be necessary: many authors have proposed that much outer disk stellar mass can be accounted for through radial migration (Sellwood \& Binney 2002; Debattista et al. 2006), which can migrate early generations of inner disk stars outward via resonances with transient spiral arms, bars, or couplings thereof (e.g., Roškar et al. 2008; Sánchez-Blázquez et al. 2009; Schönrich \& Binney 2009; Minchev et al. 2011; Roškar et al. 2012).

Because our empirical star formation laws (e.g., the conversion of $\mathrm{H} \alpha$ flux to star formation rate (SFR); Kennicutt et al. 1994) were derived in high-density environments, accounting for the fraction of stellar mass that formed in situ in outer disks assumes that these laws remain unaltered in lowdensity environments. If this is not true, conclusions drawn from typical star formation indicators about gas consumption timescales, star formation efficiency, and so on will be erroneous in outer disks and other similar environments. Consider, for example, two star-forming regions of equal mass and age, and so equal in predicted SFR. The $\mathrm{H} \alpha$ emission is sensitive to the initial mass function (IMF; e.g., Sullivan et al. 2004); hence, if one region lacks massive $\mathrm{O}$ stars, it will emit fewer ionizing photons, resulting in lower $\mathrm{H} \alpha$ flux. Measuring its SFR using a standard $\mathrm{H} \alpha-$ SFR conversion factor will thus underestimate its true SFR.

It remains an open question if star formation physics changes in low-density environments. Whether or not such a change occurs depends on whether or not changes in the underlying structure of the disk-surface mass density, gas velocity dispersion, gas phase, turbulence, etc.-affect the formation and subsequent evolution of molecular clouds and star clusters. For example, Meurer et al. (2009) argued that the formation of dense bound clusters is inhibited in regions of low mass surface density because the midplane pressure in the disk influences internal cloud pressures (see, e.g., Dopita \& Sutherland 2003). If massive stars form via competitive accretion (Larson 1973), in which interactions between protostars drive mass segregation and subsequent gas accretion in high-density cluster cores, protostars in low-density clusters would suffer fewer 
interactions and accrete less mass, inhibiting the growth of high-mass stars (e.g., Bonnell et al. 2004). Seeking out changes to the IMF in populations of young clusters could thus help determine how sensitive star formation within dense cores and molecular clouds is to the surrounding environment.

Some evidence does indicate that the cloud-to-cloud physics of star formation may be influenced by the local surface density of the disk. In inner disks, star formation follows a power law of the form $\Sigma_{\mathrm{SFR}} \propto \Sigma_{\text {gas }}^{\alpha}$, with the measured value of $\alpha$ ranging between $\sim 1$ and 1.5 (as originally proposed by Schmidt 1959 and subsequently confirmed observationally, e.g., Kennicutt 1989, 1998; Kennicutt et al. 2007; Bigiel et al. 2008). Such studies have been much rarer in outer disks and other low-density environments, partly because of a lack of $\mathrm{CO}$ emission (likely due to low metallicity or changes in ISM pressure; Elmegreen \& Hunter 2015). However, those that have broached this regime find a significantly steeper value of $\alpha$ ( 2-3; Bigiel et al. 2008, 2010; Bolatto et al. 2011; Schruba et al. 2011), implying a significantly less efficient coupling between star formation and gas density than that found in the inner disk.

Clues to this difference may come from dwarf irregular (dIrr) or LSB galaxies, which, like outer disks, are often gasdominated and low in mass surface density (McGaugh \& de Blok 1997; van Zee et al. 1997; Hunter et al. 2011). Stellar and gaseous disks in dIrr galaxies are also thicker than normal spirals (Elmegreen \& Hunter 2015), which can help stabilize them (Vandervoort 1970); outer disks may again be similar, as they are frequently warped (Sancisi 1976; Bottema et al. 1987; van der Kruit 1987; García-Ruiz et al. 2002; van Eymeren et al. 2011). In a case study of the dIrr Sextans A, Hunter \& Plummer (1996) found that stars still form at a slow rate in the peaks of the gas distribution even though dynamical arguments suggest this should not be the case (e.g., Toomre 1964; Kennicutt 1989). Van Zee et al. (1997) found similar results for six additional LSB dwarf galaxies. These galaxies lack interaction signatures; hence, van Zee et al. (1997) proposed that star formation therein is likely regulated by feedback, such as stellar winds or supernovae, locally compressing gas. Such a mechanism may be necessary to sustain star formation in environments that lack the periodic forcing provided by spiral arms or bars, which may also be absent in outer disks (Watkins et al. 2016).

One might thus consider whether these differing mechanisms yield observationally distinct populations of young clusters and $\mathrm{H}$ II regions. This is currently a topic of considerable discussion, and some previous studies have uncovered hints to this effect. Hoversten \& Glazebrook (2008), for example, found that the integrated colors of dwarf and LSB galaxies suggest a deficiency in high-mass stars; this may be related to their low integrated SFRs (Gunawardhana et al. 2011). A lack of high-mass stars may also account for the lack of highluminosity $\mathrm{H}$ II regions in dwarfs and LSB galaxies (Helmboldt et al. 2005, 2009). Yet Schombert et al. (2013) found that when all 54 LSB galaxies in their sample were taken as a whole, the $\mathrm{H}$ II region luminosity function (LF) was the same as that found in normal spirals. Hence, the lack of bright H II regions in LSB galaxies could be merely a sampling effect, given the intrinsic rareness of high-luminosity $\mathrm{H}$ II regions in general.

One means of informing this debate is to compare and contrast different star formation tracers. The SFR conversion factors assume the following: that stars are sampled from a universal IMF, that the SFH is constant over Gyr timescales, and that there is no attenuation by dust (Kennicutt 1983; Donas et al. 1987). Under those assumptions, different SF indicators should yield identical SFRs. Conversely, if different SF indicators yield different SFRs, one or more of those assumptions must be invalid. For example, when properly accounting for dust, $\mathrm{H} \alpha$ emission traces mainly $\mathrm{O}$ stars with masses $M_{*} \gtrsim 10 M_{\odot}$, while far-ultraviolet (FUV) emission traces $\mathrm{O}$ and $\mathrm{B}$ stars down to $M_{*} \sim 3 M_{\odot}$ (Kennicutt \& Evans 2012); hence, variation in the $\mathrm{H} \alpha$-to-FUV flux ratio (hereafter $\left.F_{\mathrm{H} \alpha} / F_{\mathrm{FUV}}\right)$ can be used to study the behavior of the high-mass end of the IMF in young clusters (e.g., Lee et al. 2009).

This ratio also shows trends that may hint at environmentally dependent star formation physics: globally averaged $F_{\mathrm{H} \alpha} / F_{\mathrm{FUV}}$ correlates with galaxy stellar mass (Boselli et al. 2009; Lee et al. 2009), $R$-band surface brightness (Meurer et al. 2009, but see Weisz et al. 2012), and radius in some galaxies (Thilker et al. 2005; Goddard et al. 2010; Hunter et al. 2010). Unfortunately, $F_{\mathrm{H} \alpha} / F_{\mathrm{FUV}}$ is sensitive to a large number of variables, which makes interpretation of these trends difficult. In addition to dust extinction (in fact, $F_{\mathrm{H} \alpha} / F_{\mathrm{FUV}}$ correlates extremely well with extinction, to the point that it can itself be used as an extinction estimator; Cortese et al. 2006; Koyama et al. 2015), $F_{\mathrm{H} \alpha} / F_{\mathrm{FUV}}$ decreases rapidly with age (e.g., Leroy et al. 2012) as the high-mass stars traced by $\mathrm{H} \alpha$ emission die off. IMF sampling effects play a similar role and introduce stochasticity in $\mathrm{H} \alpha$ emission at low $\mathrm{H}$ II region mass, where a given $\mathrm{H}$ II region may be powered by a single O or B star (Lee et al. 2009, 2011). These degeneracies have led to much discussion regarding the true origin of the observed $F_{\mathrm{H} \alpha} / F_{\mathrm{FUV}}$ trends, with explanations ranging from a changing IMF at low density (Pflamm-Altenburg \& Kroupa 2008; Meurer et al. 2009; Pflamm-Altenburg et al. 2009), to age effects (Alberts et al. 2011), to stochastic sampling (Goddard et al. 2010; Hermanowicz et al. 2013) or nonuniform SFHs (Weisz et al. 2012).

The nearby face-on spiral M101 (NGC 5457) provides a unique target for investigating the connection between star formation and local environment. Broadband imaging by Mihos et al. (2013) found extremely blue $(B-V \sim 0.2-0.4)$ colors in the extended LSB outer disk of the galaxy, implying a significant population of young stars at large radius. This is also apparent from deep GALEX FUV and near-ultraviolet (NUV) imaging, which show that the galaxy has an extendedultraviolet (XUV) disk (Thilker et al. 2007). Given its disturbed morphology, this extended star formation likely resulted from an interaction with one or both of its companions, NGC 5477 and NGC 5474 (Mihos et al. 2013). Both companions are star-forming themselves and nearby on the sky. The M101 galaxy group thus provides examples of three different kinds of star-forming environments in close proximity; a high-mass star-forming disk, an LSB star-forming outer disk, and two star-forming companion galaxies with lower mass.

As such, we targeted the M101 Group for deep narrowband $\mathrm{H} \alpha$ imaging with the Burrell Schmidt Telescope at Kitt Peak National Observatory (KPNO). The Burrell Schmidt's wide field of view allows for a direct comparison of all three galaxies in the M101 Group in a single mosaic image. We use our $\mathrm{H} \alpha$ narrowband imaging data in conjunction with the deepest available GALEX FUV and NUV images of M101 and its companions in order to investigate the statistical properties of the $F_{\mathrm{H} \alpha} / F_{\mathrm{FUV}}$ ratio in both of the $\mathrm{H}$ II regions as a function of 
these three environments. In Section 2, we give a brief overview of our observation and data reduction procedures. In Section 3, we describe our methodology for analyzing the H II regions, including extinction correction, H II region selection, and photometry. We present the results of these analyses in Section 4. In Section 5, we discuss the implications of our results in the context of previous analyses of the $F_{\mathrm{H} \alpha} / F_{\mathrm{FUV}}$ ratios of galaxies, as well as the broader applicability of our results. We conclude with a summary in Section 6.

\section{Observations and Data Reduction}

Here we present a discussion of our observing strategy and data reduction techniques. We briefly review these here; for an exhaustive description, we refer the reader to our previous work (Watkins et al. 2014; Mihos et al. 2017 and references therein). However, this previous work used broadband filters; hence, we focus in this section on adjustments to the procedures that are necessary in shifting to narrowband imaging data.

\subsection{Observations}

We observed M101 with the Burrell Schmidt telescope at KPNO in the spring of 2014 using two custom narrowband interference filters. The two filters have central wavelengths at 6589 and $6726 \AA$ (hereafter the on-band and off-band filters, respectively) with $\sim 100 \AA$ widths, necessitated by the Schmidt's fast $f / 3.5$ beam. The on-band filter covers $\mathrm{H} \alpha$ at M101's velocity ( $240 \mathrm{~km} \mathrm{~s}^{-1}$; de Vaucouleurs et al. 1991), while the off-band filter covers the adjacent stellar continuum. Given M101's low inclination, all $\mathrm{H} \alpha$ emission from the galaxy lies within a region of the on-band filter with $\sim 96 \%$ transmission. The on-band filter bandpass is wide enough to include Milky Way emission; however, M101 is located at a high Galactic latitude in a field relatively free of Galactic cirrus (Schlegel et al. 1998; Schlafly \& Finkbeiner 2011), limiting contamination. We observed only on moonless, photometric nights using exposure times of $1200 \mathrm{~s}$ for both filters, with dithers of $\sim 0.5$ between exposures to remove large-scale artifacts such as flat-fielding errors and scattered light. This resulted in sky levels of 200-300 ADU in the on-band filter and 150-250 ADU in the off-band. In total, we observed M101 in each filter for $71 \times 1200 \mathrm{~s}$ (nearly $24 \mathrm{hr}$ per filter).

Due to low sky counts in the narrowband filters, we could not construct flats from night-sky frames alone. To construct the flats, we started with twilight exposures; however, given our large field of view, these twilight flats contained noticeable gradients induced by the setting Sun. We therefore also produced flats without gradients using offset night-sky frames with exposure times equal to our object frames (1200 s for both filters), as we did in constructing flat fields for our broadband imaging (see Watkins et al. 2014; Mihos et al. 2017). The final twilight flats consisted of $\sim 110$ individual exposures per filter, averaging $\sim 20,000 \mathrm{ADU}_{\text {pixel }^{-1}}$, while final night-sky flats totaled $82 \times 1200 \mathrm{~s}$ exposures in the on-band and $74 \times 1200 \mathrm{~s}$ exposures in the off-band. We defer a discussion of how we used both of these flats for the final reduction to the next section.

Finally, we observed spectrophotometric standard stars from Massey et al. (1988) for photometric calibration, along with several $1200 \mathrm{~s}$ exposures of Arcturus in order to model internal reflections and the extended wings of the Schmidt point-spread function (PSF; see Slater et al. 2009).

\subsection{Data Reduction}

We began data reduction by applying a standard overscan and bias subtraction, correcting for nonlinear chip response, and applying a world coordinate system (WCS) to each frame.

Flat-fielding took place in stages. We first constructed master twilight flats by median-combining all $\sim 110$ twilight exposures per filter. To remove gradients in the twilight flats, we then constructed night-sky flats as described in previous works (Watkins et al. 2014; Mihos et al. 2017). In short, for each frame, we created an initial mask using the IRAF $^{3}$ task objmask, hand-masked any remaining artifacts (typically light scattered by stars just off-frame), and combined the resulting masked frames into a preliminary flat. We then flattened and sky-subtracted all night-sky frames using this preliminary flat, combined the flattened and sky-subtracted images into a new flat, and repeated for five iterations, until the flat field converged.

We isolated the twilight-flat gradients through division by the gradientless night-sky flats. We then modeled and divided the planes out of the twilight flats, resulting in final generation flat fields. This is mathematically equivalent to using the nightsky flats (modulo uncertainty in the gradient fits) but with the improved Poisson statistics of the twilight flats on small scales.

Mild fringing is visible in all of our on-band images at an amplitude of $\sim 0.1 \%$, but it is absent in the off-band images. As M101 is far from the ecliptic plane (hence from zodiacal light contributions), the main contributor of this fringing is telluric emission lines (OH; Massey \& Foltz 2000), which are not present in the off-band filter. We thus measured and corrected for fringing in on-band frames only. Because scattered sunlight dominates the telluric emission in the twilight frames, the twilight flats lack the fringe pattern. Hence, to isolate the pattern, we divided the night-sky flat (which does contain the pattern) by the twilight. We then scaled a normalized version of this fringe map to the sky level of each on-band frame (corrected for large-scale gradients) and subtracted it from each frame. Because this fringing is present on all onband night-sky frames, we reconstructed the on-band night-sky flat after fringe removal and rederived the on-band twilight-flat gradient before flat-fielding the on-band object frames.

For our final flux calibration, we observed spectrophotometric standard stars from the Massey et al. (1988) catalog. We derived photometric zero points by convolving our filter transmission curves over the spectra of these stars to derive filter magnitudes (defined as $-2.5 \log \left(F_{\text {filt }}\right)$ for simplicity, where $F_{\text {filt }}$ is the total flux in ADU of the star through the filter), which we compared with instrumental magnitudes derived through photometry of each exposure of each star. In each observing run, we observed 12 unique standard stars, several of which we observed multiple times to improve the final zero points. Due to uncooperative weather, we did not achieve adequate airmass coverage from these standard-star observations; instead, we derived airmass terms for each filter using the photometry of Sloan Digital Sky Survey (SDSS, DR8; Aihara et al. 2011) stars found in the individual exposures of M101

\footnotetext{
3 IRAF is distributed by the National Optical Astronomy Observatory, which is operated by the Association of Universities for Research in Astronomy (AURA), Inc., under cooperative agreement with the National Science Foundation.
} 
(this is described in more detail below). The photometric zero points are thus simply

$$
Z P=-2.5 \log \left(F_{\text {filt }}\right)-\left(m_{\text {inst }}-\kappa \sec z\right),
$$

where $m_{\text {inst }}$ is the instrumental magnitude and $\kappa$ is the airmass term.

For each filter, we take as the zero point the median value of the zero points derived from each star. The standard error on the median is $1.253 \sigma / \sqrt{N}$; hence, the errors on the two filter zero points are $\sigma_{\text {on }}=0.006$ and $\sigma_{\text {off }}=0.003$ mag. This translates to an error of $\sim 2 \%$ on M101's total flux. In our final mosaic of M101, $1 \mathrm{ADU}$ pixel $^{-1}$ per $1200 \mathrm{~s}$ is equal to an $\mathrm{H} \alpha$ surface brightness of $\Sigma_{\mathrm{H} \alpha}=3.557 \times 10^{-18} \mathrm{erg} \mathrm{s}^{-1} \mathrm{~cm}^{-2}$ $\operatorname{arcsec}^{-2}$, or an emission measure (EM) of $\sim 1.78 \mathrm{~cm}^{-6} \mathrm{pc}$. Using this flux calibration, we find good agreement (to within $\sim 3 \%)$ with the value of M101's total flux published by Kennicutt et al. (2008), measured within their value of $R_{25}$.

To reduce scattered light artifacts, we also remove reflections and diffuse halos around bright stars in all frames in the manner described by Slater et al. (2009). Briefly, we use deep (1200 s) exposures of Arcturus at different positions on the chip to measure and model these reflections and halos, then we scale and subtract them from all stars brighter than $V=10.5$ found in each frame. We do this scaling via a rough photometric calibration using SDSS stars found in each field, assuming our on-band filter is equivalent to SDSS $r$ with no color term. This produces fairly robust scalings for the reflection- and halo-subtraction process; only for the brightest stars $(V>8)$ do we need to tweak the derived magnitudes by hand in order to produce an acceptable subtraction. Given this stability, the large number of SDSS stars in each frame, and the improved airmass coverage, we choose to use the airmass terms derived in this way over those derived from the standard-star exposures for our flux calibration. This choice has little effect on the calibration, as the airmass terms are quite small $(\lesssim 0.1)$ for both filters.

Finally, we sky-subtract each frame by masking all bright stars and galaxies, fitting sky planes to each masked image, and subtracting these planes from the frames. To preserve precise flux scaling, we then scale these images to zero airmass and median-combine them into two final mosaics (an on-band and off-band) using the IRAF tasks wregister and imcombine. Because these two mosaics combine many exposures taken under variable observing conditions, a direct subtraction of the two does not produce a clean difference image, making it difficult to identify LSB regions. Hence, we create a third mosaic using individual pairs of images taken back-to-back. We align both images to within 0.1 pixel, photometrically scale and subtract the off-band images from the on-band, and combine the individual difference images into one mosaic, as before. While we use this difference mosaic to display our data, all $\mathrm{H} \alpha$ fluxes quoted henceforth are measured from the on-band and off-band mosaics, which preserve the flux calibration most accurately.

In our previous work, the background sky scatter was dominated by unresolved sources (background galaxies and foreground stars; see Rudick et al. 2010); however, the grand majority of these sources have no emission lines that fall within our two narrowband filters and thus cleanly subtract out. This, combined with our large total exposure time, results in extremely low background noise. We calculate the background sky uncertainty as the dispersion in the median count levels measured in 50-100 blank apertures with radius 15 pixels (22") chosen adjacent to the target galaxies. Near M101, the background scatter in the difference image is $\sigma \sim 0.15 \mathrm{ADU}$, giving a limiting depth of $\Sigma_{\mathrm{H} \alpha, \mathrm{lim}} \sim 5.34 \times 10^{-19} \mathrm{erg} \mathrm{s}^{-1} \mathrm{~cm}^{-2} \operatorname{arcsec}^{-2}(\mathrm{EM} \sim 0.27)$. The scatter is slightly lower near NGC $5474(\sigma \sim 0.13$ ADU), despite it being nearer the edge of the mosaic; this is due to the presence of several slightly imperfectly subtracted reflections from bright stars near M101.

Figure 1 shows a subset of our full difference mosaic, with several areas of interest zoomed in to showcase the wealth of LSB H $\alpha$ emission we detect. We also tentatively identify an extremely extended LSB plume of $\mathrm{H} \alpha$-emitting gas northeast of M101. While it is barely visible in Figure 1, we show an enhanced image of it in Figure 2, which shows our difference image masked of bright pixels (masks shown in white) and median-binned into $9 \times 9$ pixel bins.

The plume spans a length of $\sim 30 \mathrm{kpc}$ and has a characteristic surface brightness of $\Sigma_{\mathrm{H} \alpha}=1.4 \times 10^{-18}(\mathrm{EM} \sim 0.7)$ extending from the diffuse star-forming northeast plume region discussed by Mihos et al. (2013). When compared to adjacent background regions of similar size and shape (see Rudick et al. 2010; Watkins et al. 2014), this surface brightness amounts to roughly a $2 \sigma$ detection. So well removed from M101's star-forming disk, the ionization source for this plume is unclear. One possibility is that it is gas ionized by the metagalactic ionizing background; however, the feature's $\mathrm{H} \alpha$ surface brightness is roughly an order of magnitude higher than expected for this phenomenon (Vogel et al. 1995). Additionally, we see no evidence of diffuse ionized gas (DIG; see, e.g., Reynolds 1990; Haffner et al. 2009) in the long, low column density $\mathrm{H}$ I feature on the opposite, southwest side of M101 (Mihos et al. 2012), as might be expected if the ionization was from the metagalactic background. A more mundane explanation might be that the plume is diffuse $\mathrm{H} \alpha$ located within our own Milky Way galaxy. The velocity width of our filter also covers Galactic ISM velocities, and an examination of the H I data cube of Mihos et al. (2012) shows copious diffuse Galactic H I projected across the M101 Group. If this Milky Way gas is ionized, it would show as a patchy screen of diffuse $\mathrm{H} \alpha$ across our image. However, the spatial coincidence of the $\mathrm{H} \alpha$ tail with the northeast plume in M101's tidally distorted outer disk, as well as the lack of any comparable features elsewhere in our mosaic (which covers $2^{\circ} \times 2^{\circ}$ ), remains intriguing.

\subsection{GALEX Data}

In order to measure the $F_{\mathrm{H} \alpha} / F_{\mathrm{FUV}}$ ratio, as well as to correct for extinction, we use the deepest available GALEX FUV and NUV images of M101 and its companion, NGC 5474. The images of M101 were taken as part of the guest observing program in 2008 (GI3_05) and were first published in Bigiel et al. (2010). These images have exposure times of $\sim 13,300 \mathrm{~s}$ in both FUV and NUV. The images of NGC 5474 were taken as part of the Nearby Galaxy Survey (NGS; Bianchi et al. 2003) and have exposure times of $1610 \mathrm{~s}$ in both FUV and NUV; hence, they are shallower than those of M101. We calculate all FUV and NUV fluxes directly from the intensity maps, while we calculate photometric errors on these fluxes as Poisson errors using the associated high-resolution relative response maps (as discussed in Morrissey et al. 2007). Because FUV fluxes are given as monochromatic fluxes, we multiply all 


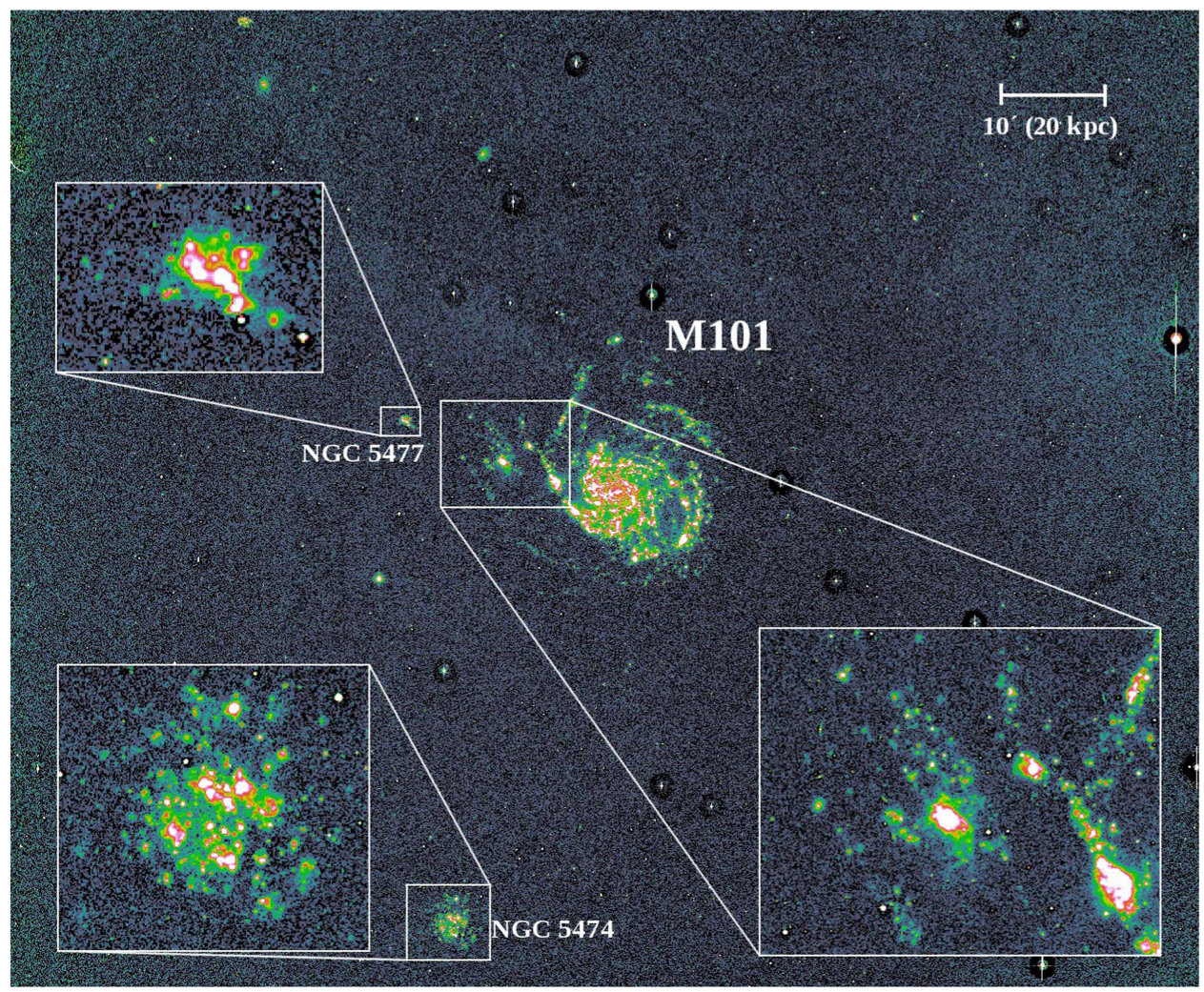

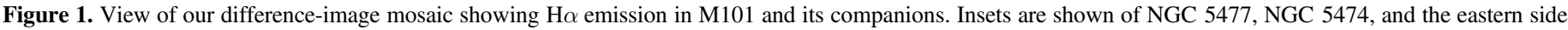

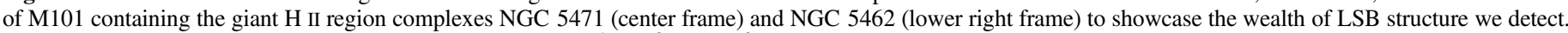
Pixels saturate (white) in this image at $\sim 2.85 \times 10^{-16} \mathrm{erg} \mathrm{s}^{-1} \mathrm{~cm}^{-2} \operatorname{arcsec}^{-2}$. North is up, and east is to the left.

FUV fluxes by the FUV filter's central wavelength in order to keep the ratio $F_{\mathrm{H} \alpha} / F_{\mathrm{FUV}}$ unitless.

\subsection{Background/Foreground Contamination}

Given the width of our filters, we detect $\mathrm{H} \alpha$ emission from sources at a large range of redshifts (we cover $\mathrm{H} \alpha$-emitting sources at $10 \%$ transmission out to $\sim 4300 \mathrm{~km} \mathrm{~s}^{-1}$ in our onband filter), resulting in both background and foreground contamination. While background spiral and elliptical galaxies are typically resolved, hence identifiable by eye, we also find many point sources in the difference mosaic that are not obviously associated with the M101 Group galaxies.

We investigated the origins of these point sources using the method described by Kellar et al. (2012). Briefly, they defined a quantity $\Delta m=m_{\mathrm{H} \alpha}-m_{R}$, where $m_{\mathrm{H} \alpha}$ is the magnitude of a source in their filters targeting $\mathrm{H} \alpha$ emission and $m_{R}$ is the magnitude of the same source in their continuum $R$-band filter, scaled such that $\Delta m=0$ for sources with no emission present in the $\mathrm{H} \alpha$ filter. They labeled unresolved sources with $\Delta m<0$ "H $\alpha$ dots," which are simply point sources that are bright in their difference images. As we use a narrowband continuum filter instead of $R$, in our case, $\Delta m=m_{\text {on }}-m_{\text {off. }}$. We utilize the same cutoff limit as Kellar et al. (2012) for "dot" selection: sources with emission-line equivalent widths $\gtrsim 30 \AA$. This corresponds to $\Delta m \lesssim-0.3$ for our filter widths of $100 \AA$.

While Kellar et al. (2012) obtained follow-up spectroscopy of the $\mathrm{H} \alpha$ bright point sources in their fields, such follow-up is beyond the scope of our project. Hence, we investigated the $\mathrm{H} \alpha$ dots in our field by cross-referencing them with SDSS and plotting their $g-r$ versus $r-i$ colors. We find that the majority of the $\mathrm{H} \alpha$ dots in our final mosaic lie in the region of color-color space occupied by $\mathrm{M}$ stars (Figure 1 of Finlator et al. 2000), while only a select few have colors bluer than this. This M-star contamination results from the width and placement of our filters. Typical M-star spectra contain broad TiO absorption features; our on-band filter's central wavelength ( $\sim 6600 \AA$ ) happens to often lie on a peak between two such features, while our off-band filter $\left(\lambda_{\text {cen }} \sim 6700 \AA\right)$ lies in an adjacent trough. This gives $M$ stars the appearance of an emission-line source in the difference mosaic.

Thankfully, these stars are readily identifiable as being bright in the difference mosaic but strongly lacking in FUV emission, as well as through available SDSS photometry. We hence reject all sources with $F_{\mathrm{H} \alpha} / F_{\mathrm{FUV}}>-1.4$ (this cutoff is also justified by Starburst99 models, which never reach $F_{\mathrm{H} \alpha} / F_{\mathrm{FUV}}$ higher than this; Leitherer et al. 1999), $g-r>1.2$, and $r-i>0.8$. The handful of dots with bluer colors are likely unresolved background galaxies, unresolved star-forming dwarfs near M101, or intergalactic H II regions (Kellar et al. 2012). For example, SDSS spectra of two of the sources show that they are quasars at $z=1.34007(\alpha=211.8225, \delta=53.75559)$ and $z=1.34536\left(\alpha=211^{\circ} .13981, \delta=53.40635\right) ;$ we detect redshifted $\mathrm{Mg}$ emission from both of these sources. These bluer sources are rare, however (we find eight across our entire field of view, for a surface density of $\sim 2 \mathrm{deg}^{-2}$ ); hence, they have a negligible effect on our analysis.

\section{Methods}

We present here our analysis of H II regions in the M101 Group. We begin by discussing our extinction-correction method. Then, we discuss how we identify $\mathrm{H}$ II regions against 


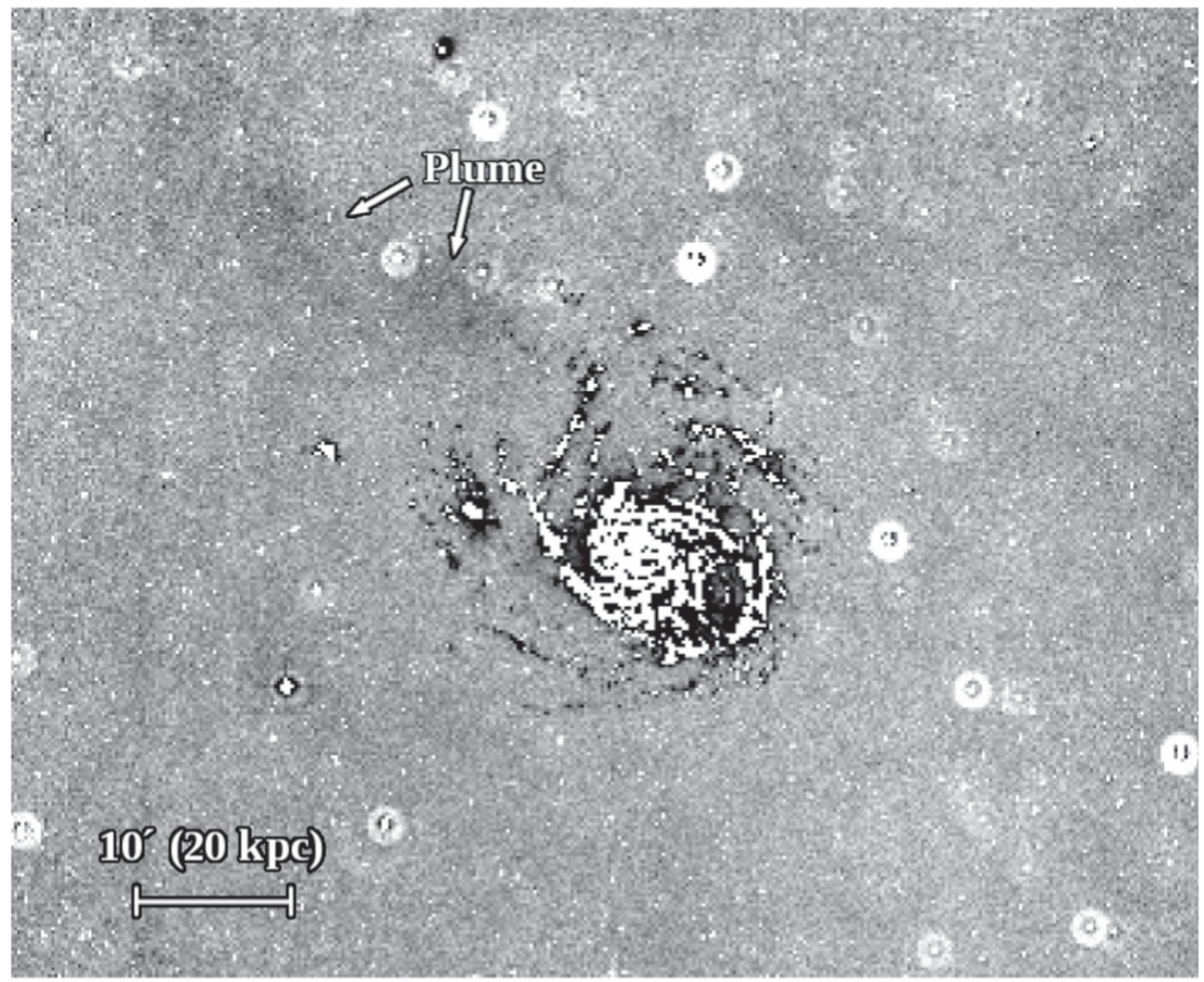

Figure 2. Masked $9 \times 9$ pixel median-binned image of our difference mosaic, showcasing a plume of extremely diffuse H $\alpha$ emission. North is up, and east is to the left.

the DIG background, and we conclude with the results of this analysis.

\subsection{Extinction Correction}

Given that we focus much of this study on the ratio $F_{\mathrm{H} \alpha} / F_{\mathrm{FUV}}$, the components of which are separated by some $\sim 5000 \mathrm{~A}$ in wavelength, some manner of extinction correction is called for. Ideally, this would be done using direct tracers of nebular extinction such as the Balmer decrement (the $\mathrm{H} \alpha / \mathrm{H} \beta$ flux ratio). While Balmer decrements have been published for $\sim 200$ of the brighter H II regions in M101 (Scowen et al. 1992), we need an extinction correction we can apply across the entire data set; so, we choose to employ the GALEXcalibrated radial IRX- $\beta$ extinction-correction method described by Goddard et al. (2010; their Section 3.6). We recap this method briefly here.

IRX- $\beta$ is an empirical relationship between the ratio of the infrared and UV luminosities (the infrared excess (IRX)) and the slope of the UV continuum $(\beta)$. It works under the assumption that all of the nonionizing UV radiation that is absorbed by intervening dust is reprocessed into the IR (Heckman et al. 1995; Meurer et al. 1995, 1999). IRX- $\beta$ can be calibrated for the GALEX passbands in the form

$$
A_{\mathrm{FUV}}=C(\mathrm{FUV}-\mathrm{NUV})+Z P,
$$

where FUV and NUV are apparent $\mathrm{AB}$ magnitudes in the respective GALEX passbands (Calzetti 2001; Seibert et al. 2005; Cortese et al. 2006; Goddard et al. 2010). For normal star-forming galaxies, Cortese et al. (2006) gave $C=5.12$, while Seibert et al. (2005) gave a value of $C=4.37$. This value depends on the assumed SFH (e.g., Calzetti et al. 2005), which affects the transformation from $\beta$ to FUV-NUV color. The value of $Z P$ depends on the age of the regions of interest and is relatively constant for populations aged between $\sim 0$ and $30 \mathrm{Myr}$ (Figure 9 in Goddard et al. 2010). We then derive the $\mathrm{H} \alpha$ extinction as $A_{\mathrm{H} \alpha}=0.5618 A_{\mathrm{FUV}}$, following Equation (13) of Calzetti (2001).

Following Goddard et al. (2010), we make bulk radial extinction corrections using the median FUV-NUV color of the $\mathrm{H}$ II regions (hence excluding DIG and field $\mathrm{O}$ and $\mathrm{B}$ stars) in both M101 and NGC 5474. For ease of comparison, we adopt the same values of $C=4.82$ and $Z P=0.0$ as Goddard et al. (2010), which are, respectively, the average of the values of $C$ published in Calzetti (2001), Seibert et al. (2005), and Cortese et al. (2006) and the typical color of $\sim 10 \mathrm{Myr}$ old populations (Figure 9 in Goddard et al. 2010). We find that our results are not sensitive to these choices for reasonable values of both. The primary purpose of this correction is not to accurately account for dust effects from H II region to $\mathrm{H}$ II region but rather to make a reasonable bulk correction that places the inner and outer disks at the same mean extinction level for a more consistent comparison among environments. This is particularly pertinent in our study, in which we measure the scatter in $F_{\mathrm{H} \alpha} / F_{\mathrm{FUV}}$ from environment to environment; because we are comparing populations across large radial expanses (e.g., M101's inner versus outer disk), a strong gradient could increase the scatter in a given radial range.

For comparison, we employed an alternative correction in M101 using the extinction values published by Scowen et al. (1992), derived from the Balmer decrement. We show this comparison in Figure 3 by overplotting our UV color-derived values of $A_{\mathrm{H} \alpha}$ on the values for H II regions from Scowen et al. (1992), plotted as a 


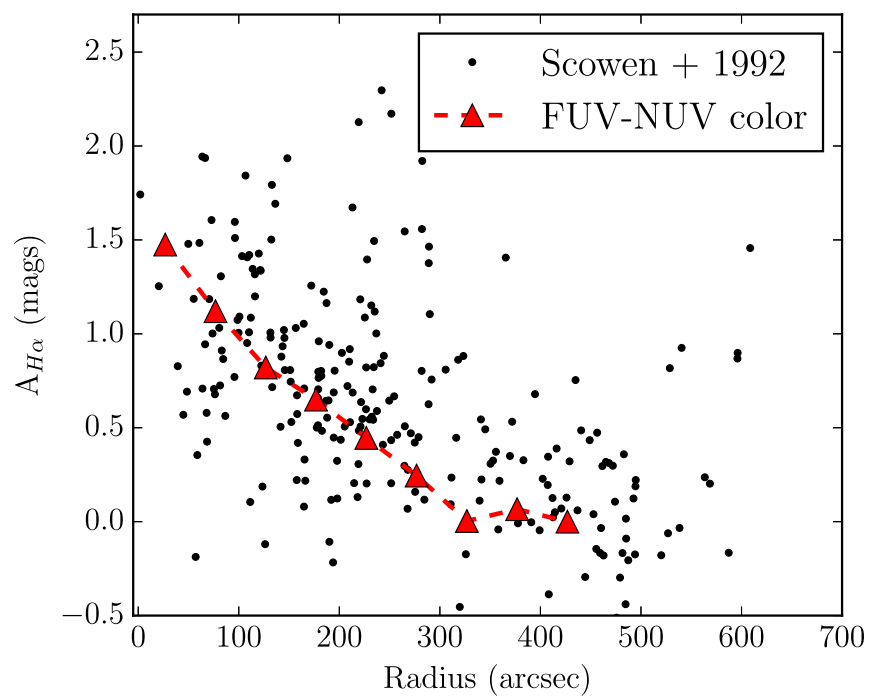

Figure 3. $\mathrm{H} \alpha$ extinction values derived from the GALEX FUV-NUV color IRX- $\beta$ relation (red triangles), compared with the extinctions of $\mathrm{H}$ II regions in M101 as derived from the Balmer decrement given by Scowen et al. (1992; black circles).

function of radius in M101. While the two are broadly consistent, the UV color-derived $A_{\mathrm{H} \alpha}$ values are consistently lower by $\sim 0.1$ mag. This is sensible, because the UV emission is directly tracing the stellar populations, which may not always lie behind a screen of dust depending on the relative dust geometry (for a beautiful demonstration of this, see Figure 1 of Whitmore et al. 2011). Because we are deriving the $\mathrm{H} \alpha$ extinction values by scaling $A_{\mathrm{FUV}}$, this geometrical uncertainty also propagates into our values of $A_{\mathrm{H} \alpha}$. However, we find through the application of both methods that this small offset does not affect the conclusions of this paper. We therefore use the UV color-derived values throughout to maintain consistency.

\subsection{Region Identification}

We use SEXtractor (Bertin \& Arnouts 1996) to identify H II regions directly from the $\mathrm{H} \alpha$ difference mosaic. Because we are selecting regions based on their $\mathrm{H} \alpha$ emission, we are focusing our study only on regions with ongoing star formation. Our interest in this particular study is in comparing physical differences in star formation (for example, changes in the IMF) across environments. Hence, by focusing on such short timescales, we avoid complications introduced by aging populations, such as the dissolution of $\mathrm{H} \alpha$-emitting regions by stellar winds (Whitmore et al. 2011).

We perform photometry on all regions using a 4." 5 (150 pc) radius aperture, which is the typical FWHM of the GALEX FUV PSF (the Burrell Schmidt PSFs in the on- and off-band images have FWHM $\sim 3^{\prime \prime}$, hence the use of the FUV FWHM is warranted). This is large enough to contain multiple $\mathrm{H} \mathrm{II}$ regions at M101's distance (see, for example, Quireza et al. 2006 for sizes of Milky Way H II regions); we discuss how this affects our conclusions in Section 4.3. However, our statistical analyses are also robust to moderate adjustments to the aperture size. Additionally, we apply an aperture correction of 0.247 mag to the FUV fluxes, derived from FUV-bright stars in the M101 field (this agrees well with the curve of growth presented by Morrissey et al. 2007).
To efficiently pick out both outer disk and inner disk H II regions, we run SEXtractor at a $2 \sigma$ threshold on an unsharpmasked version of our difference mosaic without deblending. This turns SEXtractor into something of a local peak-finding algorithm and hence is useful for identifying the often densely packed inner disk H II regions against the smooth background DIG. That said, it results in many spurious detections; thus, we employ several rejection criteria. First, we run SEXtractor in dual-image mode, measuring the fluxes of difference-image detections from the FUV images. We reject all regions with $F_{\mathrm{FUV}} \leqslant \sigma_{\text {sky,FUV }}$, where $\sigma_{\text {sky,FUV }}$ is the pixel-to-pixel background dispersion in the FUV images (measured from the intensity maps in the manner described in Section 2.2). We also reject sources with $F_{\mathrm{H} \alpha} / F_{\mathrm{FUV}}>-1.2$, which is set by the maximum $F_{\mathrm{H} \alpha} / F_{\mathrm{FUV}}$ value we find in Starburst99, from a zeroage cluster with $1 / 50$ solar metallicity lower than the lowest metallicity found in M101 (Croxall et al. 2016). We also reject sources with $g-r>1.2$ and $r-i>0.8$ to remove M stars (Section 2.4). Finally, we reject all sources $>1440^{\prime \prime}$ (48 kpc) in radius from $\mathrm{M} 101$ and $>360^{\prime \prime}(12 \mathrm{kpc})$ in radius from NGC 5474.

These cuts remove the bulk of the contaminating sources. However, running SEXtractor with no deblending detects not only $\mathrm{H}$ II regions but also local peaks in the DIG. These regions are identifiable by eye as being more uniform in flux across the photometry aperture (as opposed to the point source-like H II regions). However, to reduce subjectivity, we make a firstround rejection of such regions via an automated procedure. We define a concentration parameter

$$
c_{50}=1-f_{\mathrm{px}, 50},
$$

where $f_{\mathrm{px}, 50}$ is the fraction of pixels in the photometry aperture containing $50 \%$ of the total flux ( $c_{50}$ is defined such that high values correspond to higher concentration). We iterate the threshold value of $c_{50}$ until we see a reasonable rejection of diffuse regions, then we reject the few remaining DIG regions by hand. We choose not to reject diffuse-looking regions in the outer disk. HII regions expand until they reach pressure equilibrium with the ISM (Dyson \& Williams 1980; GarciaSegura \& Franco 1996); hence, in low-density environments they can potentially grow quite large. The statistical analyses we discuss below are robust to this rejection procedure, as diffuse-looking regions most often have anomalously low $F_{\mathrm{H} \alpha} / F_{\mathrm{FUV}}$ (which further implies that they are mostly DIG; Hoopes et al. 2001), and are rejected as outliers in the statistical metrics we use.

One concern is that in choosing regions based on $\mathrm{H} \alpha$ emission, there is the possibility that we are missing a population of UV-bright but $\mathrm{H} \alpha$-weak clusters. This would include, for example, very massive clusters that nonetheless contain no highly ionizing, very massive stars due to a truncated IMF. We thus compare our $\mathrm{H} \alpha$-selected samples with separate samples selected from both galaxies' FUV images, using the same procedure as before. However, in this case, we reject regions based on their compactness in the FUV images, rather than the $\mathrm{H} \alpha$ image, in order to preserve FUV-emitting clusters. While the FUV-selected samples did uncover a large ( 200) number of additional very FUV-faint regions (mostly in the outer disk) as compared to the $\mathrm{H} \alpha$-selected sample, we find no significant number of additional FUV-bright regions at any radius. All of the additional FUV-faint regions selected 
M101

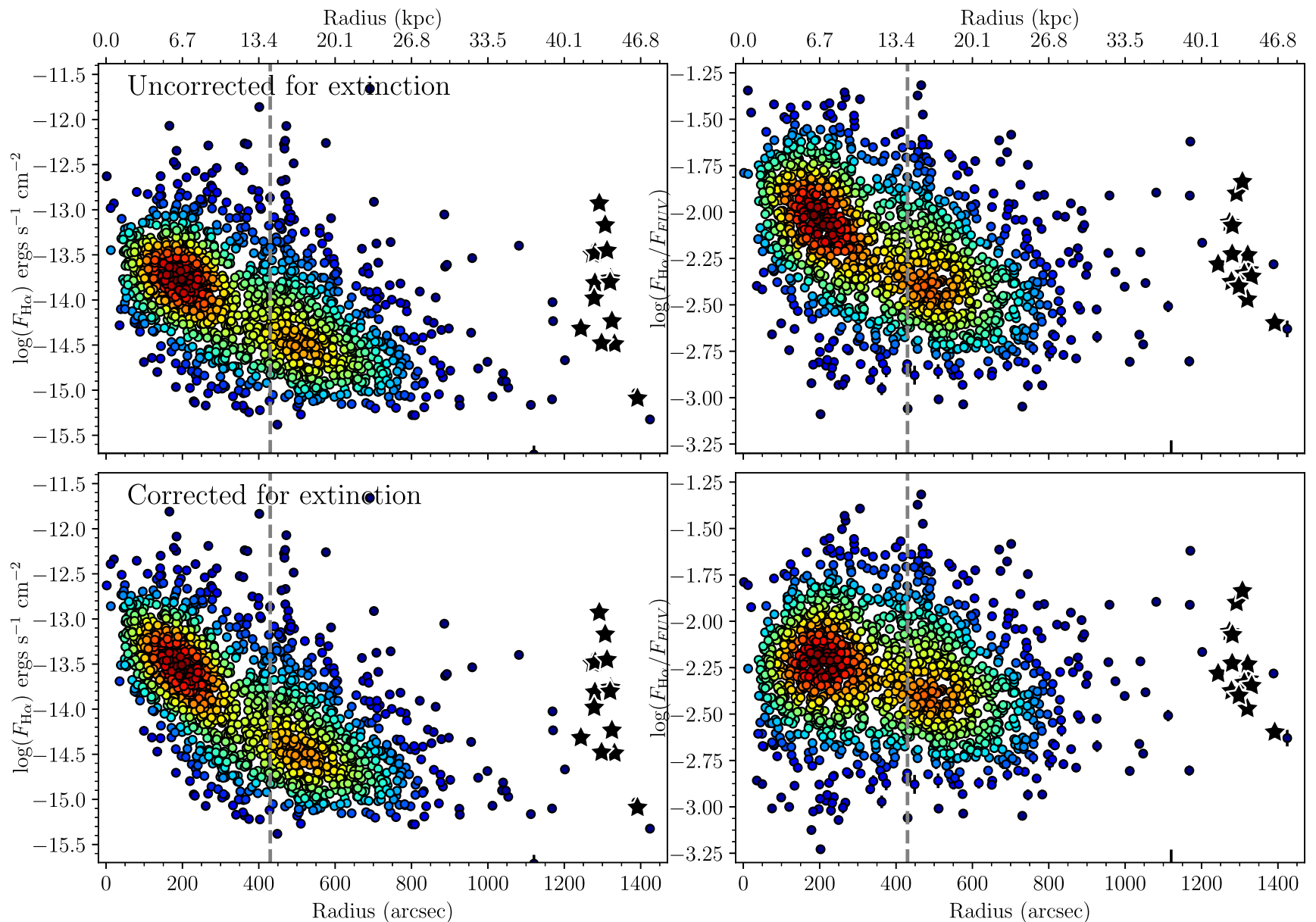

Figure 4. Left: $\mathrm{H} \alpha$ fluxes of M101 H II regions plotted against radius. The top panels show fluxes uncorrected for extinction, while the bottom panels show fluxes after the correction described in Section 3.1 is applied. The colors represent the local density of points in the plot. Black stars represent regions located within the dwarf companion NGC 5477. The gray dashed lines show our chosen inner disk-outer disk demarcation. Right: $F_{\mathrm{H} \alpha} / F_{\mathrm{FUV}}$ of all M101 H II regions plotted against radius. Symbol colors and types and the gray dashed line are the same as in the left panels.

also cover a wide range of $\mathrm{H} \alpha$ flux $\left(\log \left(F_{\mathrm{H} \alpha}\right) \sim-14.6\right.$ to $-17 \mathrm{erg} \mathrm{s}^{-1} \mathrm{~cm}^{-2}$ ), and most appear diffuse and irregular in the $\mathrm{H} \alpha$ difference image. For example, many lie in the diffuse outskirts of $\mathrm{H}$ II region complexes or along filaments of more isolated diffuse emission. This implies that they could mostly be older FUV-emitting clusters embedded within the DIG. It thus appears that if there is a population of FUV-bright but $\mathrm{H} \alpha$-faint regions in either M101 or NGC 5474, it is not significant with respect to the general population of starforming regions in either galaxy at any radius.

\section{Results}

\subsection{H II Region Photometry}

We show the results of our $\mathrm{H}$ II region photometry in Figures 4 and 5 for M101 and NGC 5474, respectively. The final sample contains $1525 \mathrm{H}$ II regions in M101 and 156 regions in NGC 5474. We show the radial profiles of $\log \left(F_{\mathrm{H} \alpha}\right)$ on the left and $\log \left(F_{\mathrm{H} \alpha} / F_{\mathrm{FUV}}\right)$ on the right. For comparison, we show the radial profiles before and after we apply the extinction correction described in Section 3.1 (top and bottom plots, respectively). The gray dashed lines in Figure 4 mark M101's outer disk, which we define as $>3$ times the azimuthally averaged disk scale length $\left(430^{\prime \prime}, 14.5 \mathrm{kpc}\right.$;
Mihos et al. 2013). In Figure 6, we show this outer disk demarcation and a potential alternative on our difference mosaic and on the $V$-band data from Mihos et al. (2013), for reference. We discuss how the choice of outer disk boundary affects our results in Section 4.2.

It should be noted here that NGC 5474 has a strongly offset bulge (van der Hulst \& Huchtmeier 1979; Kornreich et al. 1998); hence, the definition of its "center" is not entirely clear. We define its center as the centroid of the circular outer isophotes (at $180^{\prime \prime}$, or $6 \mathrm{kpc}$ ) on our on-band mosaic, which is very close to the kinematic center of its (strangely regular) $\mathrm{H} \mathrm{I}$ velocity field (van der Hulst \& Huchtmeier 1979). This choice does not affect the qualitative behavior of the radial profiles; however, the flux profile does show more scatter with radius when centered on the bulge. This implies that the isophotal center is the more appropriate choice regarding star formation in this galaxy.

The extinction correction has the expected behavior: $\mathrm{H} \alpha$ fluxes increase absent extinction, and $F_{\mathrm{H} \alpha} / F_{\mathrm{FUV}}$ decreases given stronger attenuation for FUV. The correction applied at all radii in the lower-metallicity companion NGC 5474 (which has a central $\mathrm{O}$ abundance of $12+\log (\mathrm{O} / \mathrm{H})=8.19$, versus 8.71 in M101; Pilyugin et al. 2014) is less severe than that applied in the dustier central regions of M101. Additionally, we 


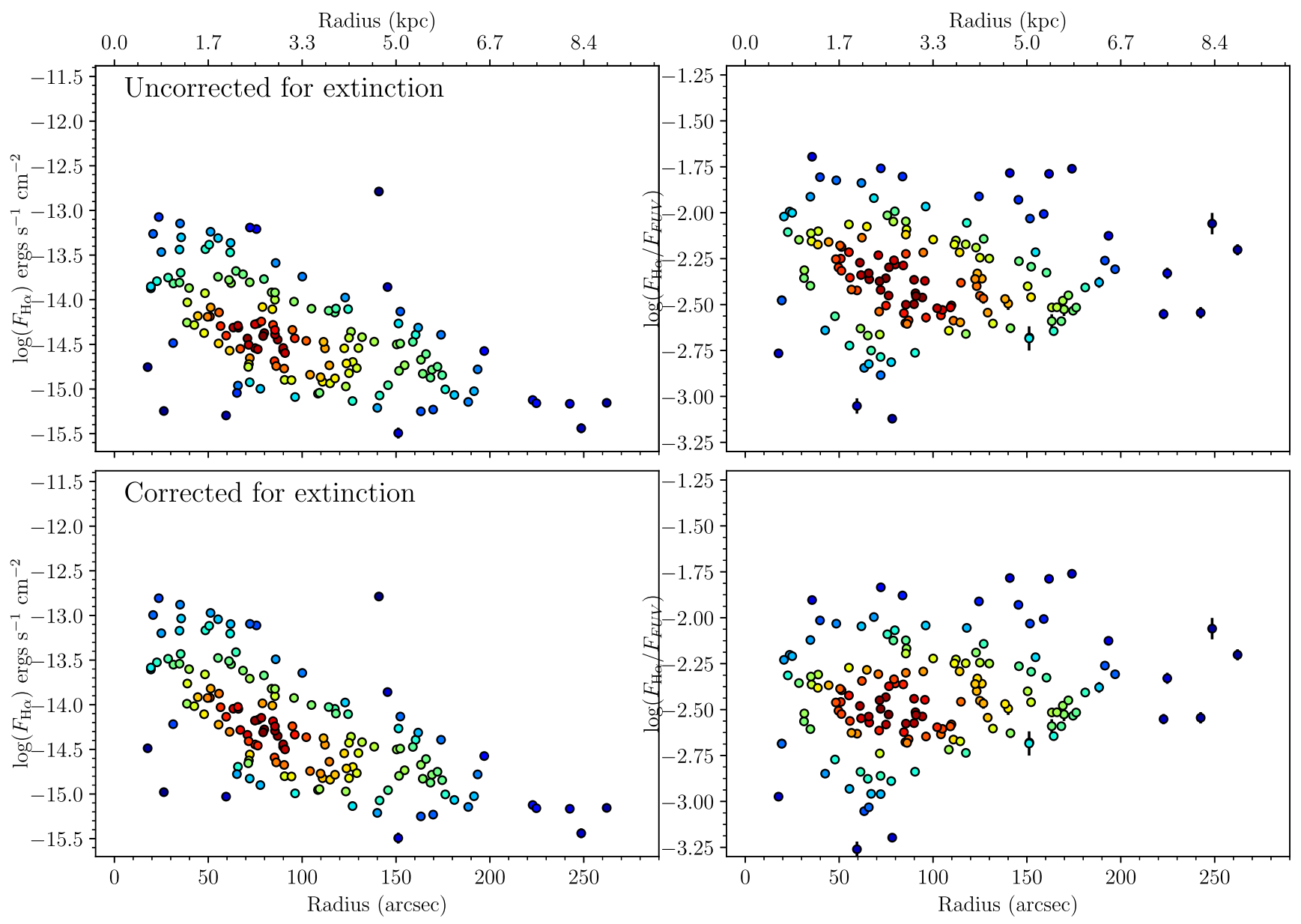

Figure 5. Same as Figure 4 but for H II regions in NGC 5474. We have used the same scale on the $y$-axes for ease of comparison.

plot the values of $\log \left(F_{\mathrm{H} \alpha}\right)$ and $\log \left(F_{\mathrm{H} \alpha} / F_{\mathrm{FUV}}\right)$ for the dIrr companion NGC 5477 (due east of M101; see Figure 1) in the same plots as M101 using black stars. Despite its much smaller mass, NGC 5477's H II regions span the same range of luminosity as those in M101's inner disk, implying similar LFs between the two environments. The same appears true of NGC 5474; we found it possible to reproduce NGC 5474's global LF by resampling from M101. Each galaxy contains pockets of high column density gas (of order $10^{21} \mathrm{~cm}^{-2}$; van der Hulst \& Huchtmeier 1979; van der Hulst et al. 2001; Walter et al. 2008), which may account for the similarity. Regardless, that all three galaxies have qualitatively similar LFs is reminiscent of the study by Schombert et al. (2013), which found that the lack of bright $\mathrm{H}$ II regions in LSB galaxies can be explained as an artifact of small-number statistics, rather than as a change in the LF itself.

Yet, though each galaxy's integrated LF appears similar, there are strong radial gradients in mean $\mathrm{H} \alpha$ luminosity in both M101 and NGC 5474. This is most likely a demonstration of the Schmidt Law: molecular gas density in M101 declines exponentially with radius (e.g., Kenney et al. 1991), hence the SFR declines accordingly (Kennicutt et al. 2007; Bigiel et al. 2008). Also, the azimuthally averaged SFR and gas density within galaxies have a power-law relationship (up to the threshold density; Kennicutt 1998); hence, it is not surprising that we see general radial declines in mean $\mathrm{H} \alpha^{-}$ux with a large region-to-region scatter. A comparison with the THINGS H I map of M101 (Walter et al. 2008) also shows that regions with the highest $\mathrm{H} \alpha$ flux for their radius always cluster around high $\mathrm{HI}$ column density peaks. That the global H II region LFs of M101 and NGC 5474 (and possibly NGC 5477) appear similar thus seems to be a consequence of each having a similar density structure within its ISM.

If gas density alone imposes the radial dependence of H $\alpha$ flux, it should affect the FUV flux in a similar way, assuming no dramatic changes in, e.g., the IMF. Indeed, Figure 4 shows that the radial gradient in $F_{\mathrm{H} \alpha} / F_{\mathrm{FUV}}$ in $\mathrm{M} 101$ is strongly reduced after the extinction correction is applied. NGC 5474 contains no strong gradient before correction; this remains mostly true after a correction is applied, although a mild positive gradient is induced, implying that perhaps we are slightly overcorrecting for extinction in this galaxy. We also find that $F_{\mathrm{H} \alpha} / F_{\mathrm{FUV}}$ and $F_{\mathrm{FUV}}$ are uncorrelated after applying an extinction correction in either galaxy. Therefore, it may be that any radial trend in mean $F_{\mathrm{H} \alpha} / F_{\mathrm{FUV}}$ in either galaxy can be attributed to extinction.

The scatter in $F_{\mathrm{H} \alpha} / F_{\mathrm{FUV}}$ also appears to be roughly constant with environment from M101's inner disk, to its outer disk, to NGC 5474, and possibly even to NGC 5477 (though with only 14 total H II regions, any measure of scatter in this galaxy will be highly uncertain). In tandem, this implies that star formation is ignorant of the global environment; other than the available 


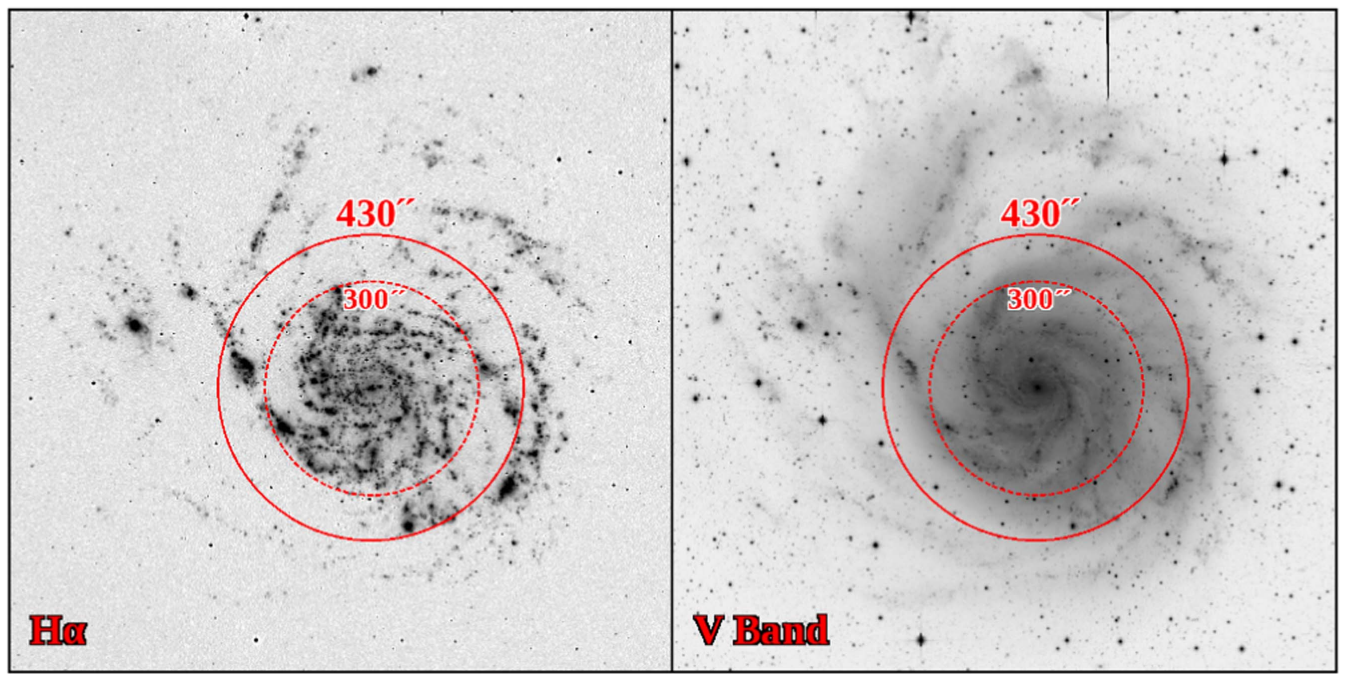

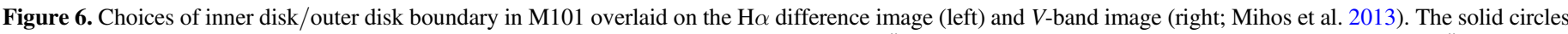

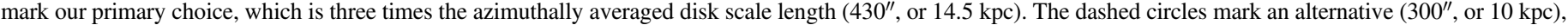
located where the $\mathrm{H} \alpha$ surface brightness profile begins to decline (Martin \& Kennicutt 2001).

fuel, it does not seem to know whether it is taking place in a low-mass galaxy, a high-density inner disk, or a low-density outer disk. We test these observations explicitly in the next section.

\subsection{Statistical Analysis}

The intrinsic $F_{\mathrm{H} \alpha} / F_{\mathrm{FUV}}$ ratio is mainly driven by the number of massive $\mathrm{O}$ and $\mathrm{B}$ stars. If present, they are the primary source of the ionizing radiation that powers the $\mathrm{H} \alpha$ emission. A truncated IMF would result in fewer massive stars being born, reducing the maximum possible $F_{\mathrm{H} \alpha} / F_{\mathrm{FUV}}$. We show this in Figure 7 via evolutionary tracks of $F_{\mathrm{H} \alpha} / F_{\mathrm{FUV}}$ in single-burst models from Starburst99 (Leitherer et al. 1999) for two different metallicities. We show both a standard Kroupa IMF (solid lines; Kroupa 2001) and a Kroupa IMF truncated at $30 M_{\odot}$ (truncations as low as $20 M_{\odot}$ have been suggested; e.g., Bruzzese et al. 2015). The tracks diverge clearly at early times ( $\lesssim 6 \mathrm{Myr}$, set by the life spans of the most massive stars), with the truncated IMF tracks peaking at much lower $F_{\mathrm{H} \alpha} / F_{\mathrm{FUV}}$, as expected. If a change in the IMF occurs at a given radius in a galaxy, the distribution of allowed values of $F_{\mathrm{H} \alpha} / F_{\mathrm{FUV}}$ in the $\mathrm{H}$ II region population will adjust accordingly. Lower variation in the region-to-region dust content in outer disks will result in a similar change once bulk radial trends are taken into account. Such behavior ought to be observable, therefore, in the scatter of bulk extinction-corrected $F_{\mathrm{H} \alpha} / F_{\mathrm{FUV}}$ within different populations of $\mathrm{H}$ II regions, assuming that variations in the median $F_{\mathrm{H} \alpha} / F_{\mathrm{FUV}}$ can be fully attributed to extinction effects.

We display the medians and two measures of the scatter in $F_{\mathrm{H} \alpha} / F_{\mathrm{FUV}}$ in Figure 8 for three regions: M101's inner disk (inside of $3 h$ ), M101's outer disk (outside of $3 h$ ), and the more massive companion NGC 5474. The inner/outer disk boundaries in M101 are marked in Figures 4 and 6 for reference.

In the top panel of Figure 8, we show box and whisker diagrams for these three regions. As a reminder, the boxes span the first through third quartiles of the data (Q1 and Q3), and the whiskers span up to $\pm 1.5 \times$ the interquartile range. Medians are shown in red, and outliers are shown as +'s. In the bottom panel, we show the values of the trimmed standard deviation $\left(\sigma_{t}\right)$ for the same three regions. This is the standard deviation of

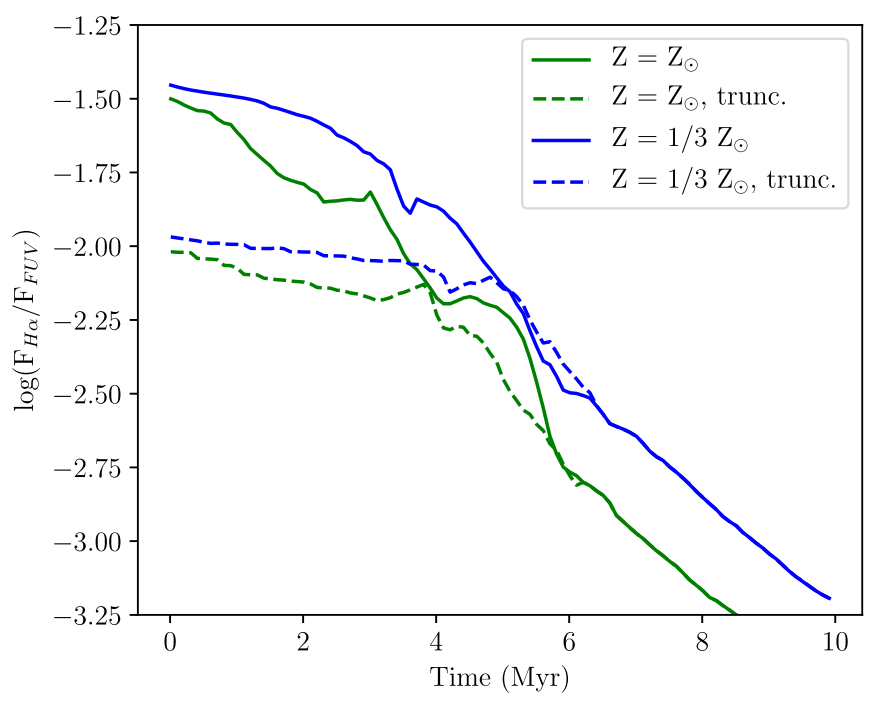

Figure 7. Starburst99 single-burst models of the time evolution of $F_{\mathrm{H} \alpha} / F_{\mathrm{FUV}}$ for two different metallicities (using Padova isochrones; Bressan et al. 2012). Solid lines show the evolution for a standard Kroupa IMF; dashed lines show a Kroupa IMF with a truncation at $30 \mathrm{M}_{\odot}$.

the sample trimmed of its top and bottom $5 \%$ of values, multiplied by a corrective factor $(1 / 0.789$ for $5 \%$ trimming; Breiman 1973; Huber 1981; Morrison et al. 1990) to ensure that $\sigma_{\mathrm{t}}$ and $\sigma$ (the standard deviation of the whole sample) are measuring the same parameter in the case of purely Gaussian data. We use $\sigma_{\mathrm{t}}$ over $\sigma$ for its robustness to outliers, such as extremely luminous $\mathrm{H}$ II regions or the handful of DIG regions that might have made it into the final sample. Other such robust estimators of scatter (such as the median absolute deviation) give similar results. The error bars on $\sigma_{\mathrm{t}}$ are simply the standard error on the standard deviation, which is equal to $1 / \sqrt{2 N}$ for sample size $N$.

After we apply our extinction correction, the median values of $F_{\mathrm{H} \alpha} / F_{\mathrm{FUV}}$ for all three regions are $-2.235 \pm 0.013$, $-2.379 \pm 0.015$, and $-2.451 \pm 0.029$, respectively. While this implies statistically significant differences in the medians from region to region, we give only the standard errors (which are equivalent to bootstrapped errors, despite the slight 


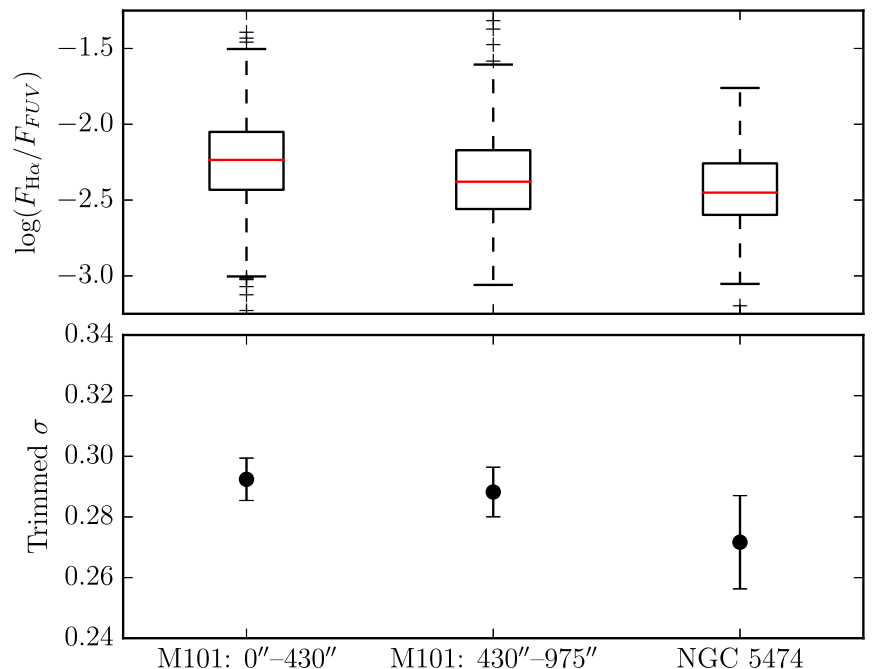

Figure 8. Top: box and whisker plots showing the distribution of $F_{\mathrm{H} \alpha} / F_{\mathrm{FUV}}$ values in three regions in the M101 Group: M101's inner disk, M101's outer disk, and NGC 5474 as a whole. Bottom: values of the trimmed standard deviation in $F_{\mathrm{H} \alpha} / F_{\mathrm{FUV}}$ for the three regions described above. Error bars are $1 / \sqrt{2 N}$ for sample size $N$. The radius $430^{\prime \prime}$ is $3 \times$ the scale length of M101.

non-Gaussianity of the data). Systematic errors on the GALEXcalibrated IRX- $\beta$ extinction correction are larger (of order 0.1 mag, excluding uncertainties in the transformation from FUV -NUV color to $\beta$; Cortese et al. 2006), which does not include the methodological uncertainty inherent in applying this correction on average in radial bins. The differences in the medians between all three regions are also smaller than the standard deviations in $F_{\mathrm{H} \alpha} / F_{\mathrm{FUV}}(\sigma \sim 0.3)$, again implying that most of the gradient in $F_{\mathrm{H} \alpha} / F_{\mathrm{FUV}}$ in M101 can likely be explained by extinction alone.

Similar box widths in Figure 8, as well as similar values of $\sigma_{\mathrm{t}}$, also suggest that the scatter in $F_{\mathrm{H} \alpha} / F_{\mathrm{FUV}}$ among the three regions is equal. We therefore compare the sample variances using Levene's test (Levene 1960). This test assesses whether or not the quantity $z_{i j}=\left|x_{i j}-\bar{x}_{i}\right|$, where $\bar{x}_{i}$ is the mean of theith group, is equal between groups. It is hence similar to the $F$-test in that it assesses the equality of variances between populations, but it is more robust to non-Gaussianity and higher in statistical power (e.g., Lim \& Loh 1996). The mean can be replaced with a more robust statistic, such as the median (e.g., Brown \& Forsythe 1974); we use the trimmed mean, defined analogously to the trimmed standard deviation.

We show the results of this test in Table 1 for the following comparisons: M101's inner disk to its outer disk, M101's inner disk to NGC 5474, M101's outer disk to NGC 5474, and all three simultaneously. Here $W$ is the value of the test statistic, while the $p$-value is defined in the standard way for confidence $1-\alpha$. In all four tests, we cannot reject the null hypothesis that the variances in $F_{\mathrm{H} \alpha} / F_{\mathrm{FUV}}$ in all three environments are equal. While, for philosophical reasons, this does not by itself prove that the variances are equal, these results in conjunction with the similarity in values of $\sigma_{\mathrm{t}}$ and widths of the box plots for each region strongly imply that this is the case. We verify that this result is not sensitive to the definition of the outer disk; the conclusion remains true for choices anywhere between $300^{\prime \prime}$ (the point at which the $\mathrm{H} \alpha$ surface brightness profile begins to decline; Martin \& Kennicutt 2001) and 600" (roughly the Holmberg radius, $R_{26.5}$; Mihos et al. 2013).
Table 1

Results of Levene's Test Trials

\begin{tabular}{lcccc}
\hline \hline Test & In-Out & In-5474 & Out-5474 & All \\
\hline$W$ & 0.293 & 0.009 & 0.052 & 0.147 \\
$p$-value & 0.588 & 0.924 & 0.821 & 0.863 \\
\hline
\end{tabular}

While the comparisons between the regions of M101 and NGC 5474 seem immune to the choice of inner disk-outer disk boundary, large uncertainties in regions with smaller sample sizes could make it harder to draw such a strong conclusion. We thus further test this through a bootstrapping experiment. For each definition of the inner disk-outer disk boundary, we randomly sample $N$ values of $F_{\mathrm{H} \alpha} / F_{\mathrm{FUV}}$ from either the inner or outer disk, with $N$ equal to NGC 5474's sample size. We then run Levene's test again between the downsampled M101 population and NGC 5474. We repeat each sampling test 10,000 times; in all tests, the resulting $p$-values are $>0.05$ between $93 \%$ and $97 \%$ of the time, providing evidence that the results of the previous tests using the full samples were not an artifact of sample size.

While these results are robust to the choice of inner diskouter disk boundary, we find that the lowest $p$-value was obtained using $300^{\prime \prime}$ rather than $430^{\prime \prime}$ ( $p=0.14$ versus 0.59 , respectively). By splitting the disk into three parts, we find that the region within $300^{\prime \prime}-430^{\prime \prime}$ does have significantly higher scatter in $F_{\mathrm{H} \alpha} / F_{\mathrm{FUV}}$. Figure 4 shows that this region has a low density of $\mathrm{H}$ II regions relative to the rest of the disk. It also appears dynamically distinct; it lies roughly at corotation with the inner disk spiral arms (Waller et al. 1997) and is the site of a severe kink in the H I rotation curve (Meidt et al. 2009). This is also the location of a pocket of high-velocity gas in the galaxy's northeast (Walter et al. 2008; Mihos et al. 2012) and a region with a high velocity dispersion (Walter et al. 2008). Dynamical effects may thus have influenced the $\mathrm{H}$ II region population in this particular area (a high gas velocity dispersion, for example, may inhibit star formation; Kennicutt 1989).

Aside from this unusual region, however, we find that once extinction is taken into account, both the median $F_{\mathrm{H} \alpha} / F_{\mathrm{FUV}}$ and the scatter in $F_{\mathrm{H} \alpha} / F_{\mathrm{FUV}}$ shows no significant variation with environment in the M101 Group. This supports our initial conjecture that, aside from gas density (which affects the intensity of the star formation), star formation on short timescales is blind to environment.

\subsection{Comparisons with Starburst99 Models}

If both the median and scatter in the $F_{\mathrm{H} \alpha} / F_{\mathrm{FUV}}$ ratio in $\mathrm{H}$ II region populations are constant with environment, once extinction is taken into account, one might question how much room is left for variations in the IMF. We explore this question through comparisons with Starburst99 (Leitherer et al. 1999) models, which we show in Figure 9.

The blue histograms in Figure 9 show the distributions of $F_{\mathrm{H} \alpha} / F_{\mathrm{FUV}}$ in $\mathrm{H}$ II regions in four radial bins within M101, uncorrected for extinction. In order to compare our data with the Starburst99 models, we choose these bins such that their mean metallicities (measured from the H II region metallicity values supplied by Scowen et al. 1992, which range from $\sim 5 \times$ solar to $\sim 1 / 5$ solar) correspond to the metallicity options available in Starburst99. While Starburst99 does not include 

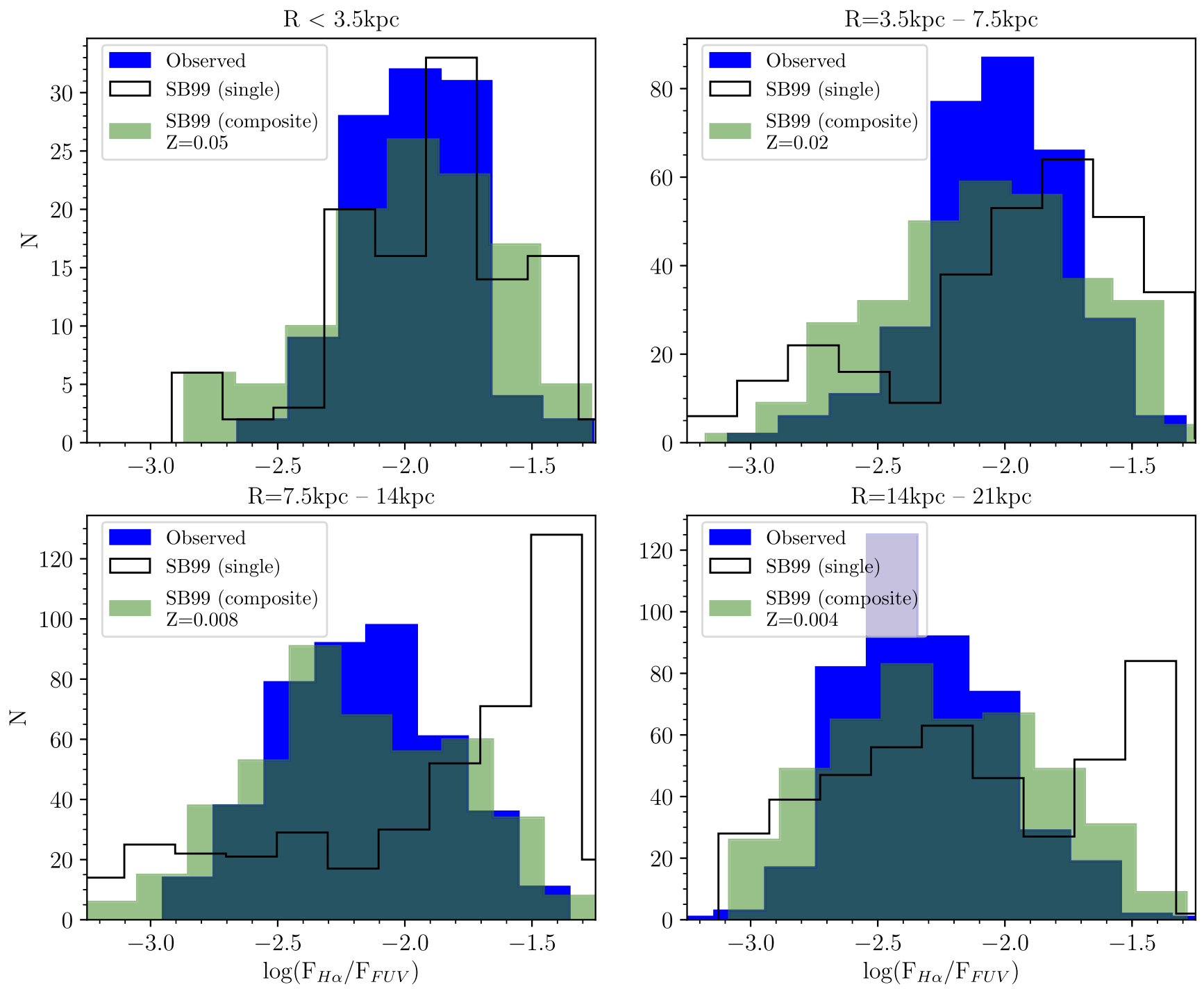

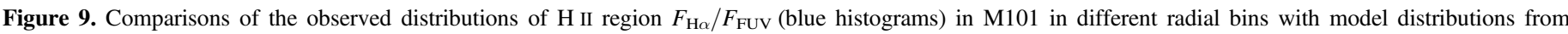

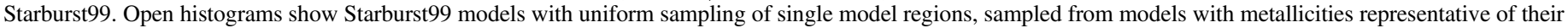
respective radial bins, while green histograms show averages of composite regions made of multiple Starburst99 models (see text).

the effects of stochastic sampling from the IMF, given our low resolution, the majority of the regions we sample are likely massive enough to be above the stochastic limit (at M101, below $\log \left(F_{\mathrm{H} \alpha}\right) \sim-15$; e.g., Hermanowicz et al. 2013); hence, these effects should not be important.

To generate model samples, we first create model evolutionary tracks of $\mathrm{H} \alpha$ flux (using the output $\mathrm{H} \alpha$ luminosity) and FUV flux (by convolving the output model spectrum at each timestep with the FUV transmission curve) normalized to unit mass for each of the four metallicities. To create a realistic cluster sample, we create a random distribution of masses following a power law with a slope of -2 (e.g., Hunter et al. 2003; Lada \& Lada 2003; Weidner et al. 2004; we note that the results are robust for any reasonable choice of slope value), uniformly sample the model fluxes in time between 0 and $10 \mathrm{Myr}$, and multiply these mass-normalized fluxes by the randomly generated cluster masses to produce a range of model cluster fluxes. We then apply extinctions to these model fluxes at random, drawn from the data set by Scowen et al. (1992) within the appropriate radial bins, and then trim the generated model regions to ensure that the distribution of model $\mathrm{H} \alpha{ }^{-}$uxes matches that of the data in each radial bin. We also reject model regions with FUV fluxes below the observational limit. The results are shown as the open histograms in Figure 9.

It can be easily seen in Figure 9 that these distributions provide a poor match to the data in all radial bins. As noted in Section 3.2, our choice of photometry aperture (4!.5) corresponds to $\sim 150 \mathrm{pc}$ at M101's distance and is hence large enough to potentially include multiple $\mathrm{H}$ II regions (as well as surrounding DIG). We verified this through visual comparison with archival HST H $\alpha$ imaging of M101 (GO13773; PI: Chandar) and found that our apertures contain typically four to five individual $\mathrm{H}$ II regions. Adjacent $\mathrm{H}$ II regions should be similar in metallicity but may not be uniform in age; the Orion Nebula complex, for example, contains four stellar associations within an $\sim 100 \mathrm{pc}$ radius that span ages from 0 to $10 \mathrm{Myr}$ (Brown et al. 1994), arguing that any individual H II region complex identified in our sample may actually consist of multiple clusters with varying ages.

Indeed, we found that we could reproduce the observed distributions much more successfully by using model clusters generated by adding together $N$ individual Starburst99 models 


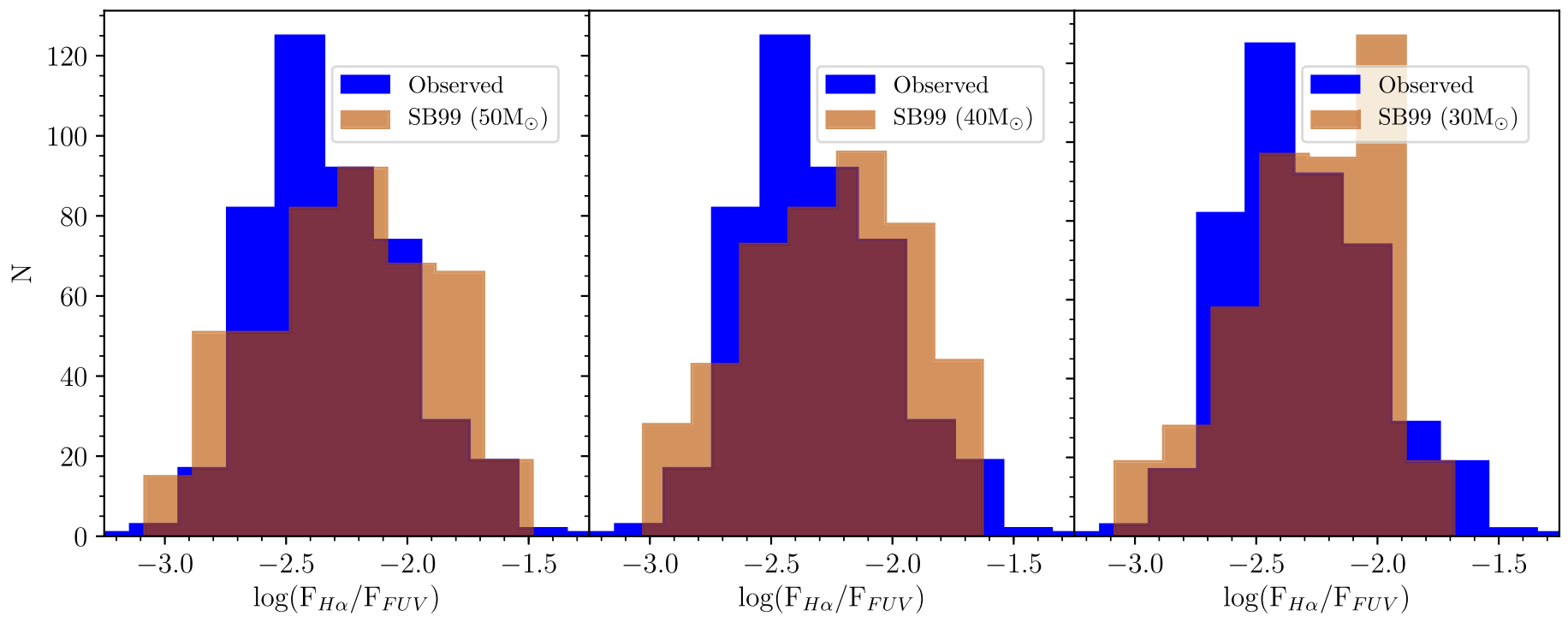

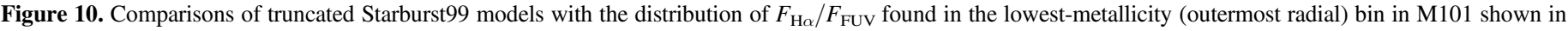

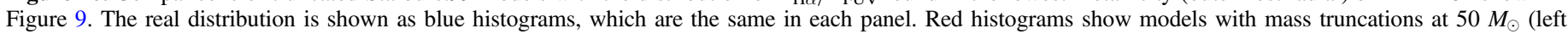
panel), $40 M_{\odot}$ (middle panel), and $30 M_{\odot}$ (right panel).

of varying ages, where $N$ is drawn from a Poissonian distribution with expectation $\lambda=4$. These are shown via the green histograms in Figure 9, where we have adopted a standard Kroupa IMF. While we cannot exactly reproduce the true distributions of $F_{\mathrm{H} \alpha} / F_{\mathrm{FUV}}$ in any radial bin, this is perhaps not surprising given the large number of assumptions we have made (single metallicities per radial bin, randomly sampled extinction values, etc.). Still, the median values of the model distributions are close to the true values (within $0.1 \mathrm{dex}$ in all radial bins), and the models tend to share the skewed Gaussian appearance of the true distributions. Given the qualitative nature of these comparisons, however, we must address two caveats.

First, we note that in order to reproduce the observed distributions in the two outermost radial bins, we must employ $\mathrm{H} \alpha$ and FUV flux cuts on both the low and high ends. We find that if we trim only low fluxes, to match our observational limits, we cannot reproduce the distributions of $F_{\mathrm{H} \alpha} / F_{\mathrm{FUV}}$ in the two outermost radial bins regardless of which IMF we choose. Pure random sampling from the cluster mass function results in too many bright clusters for these outermost regions of M101. This result is in agreement with that of PflammAltenburg et al. (2013).

Second, even with these cuts, the match appears to be poorest in the two outermost bins. As has been previously argued, because outer disks seem to lack molecular gas and show extremely inefficient star formation, the IMF in such environments may be biased toward low-mass stars (e.g., Meurer et al. 2009; Pflamm-Altenburg et al. 2009; Bigiel et al. 2010). Hence, we attempted to determine whether or not a truncated IMF provided a better match to this bin. This is shown in Figure 10, where we compare the distribution of $F_{\mathrm{H} \alpha} / F_{\mathrm{FUV}}$ values in the lowest-metallicity (outermost) radial bin to three different models, truncated at $50 M_{\odot}$ (left panel), $40 M_{\odot}$ (middle panel), and $30 M_{\odot}$ (right panel). Again, these models are averages of typically four to five model regions. We find that $50 M_{\odot}$ is the lowest truncation mass we can use in order to produce satisfactory qualitative agreement with the data. Below this mass, the model distributions of $F_{\mathrm{H} \alpha} / F_{\mathrm{FUV}}$ tend to be skewed strongly to the left and consistently lack high $F_{\mathrm{H} \alpha} / F_{\mathrm{FUV}}$ tails. The lower the truncation mass, the sharper the cutoff at high $F_{\mathrm{H} \alpha} / F_{\mathrm{FUV}}$.

Figure 7 provides an explanation: the time evolution of $F_{\mathrm{H} \alpha} / F_{\mathrm{FUV}}$ for truncated IMFs shows a plateau at early ages, the length of which depends on the lifetime of the highest-mass star, beyond which $F_{\mathrm{H} \alpha} / F_{\mathrm{FUV}}$ begins to decline. The plateau value itself also depends on the mass, such that lower truncation masses plateau at lower values of $F_{\mathrm{H} \alpha} / F_{\mathrm{FUV}}$. The standard Kroupa IMF model, by contrast, shows a steady decline over a larger range of $F_{\mathrm{H} \alpha} / F_{\mathrm{FUV}}$ values; the decay in $F_{\mathrm{H} \alpha} / F_{\mathrm{FUV}}$ reflects the larger range of contributing stellar masses, hence the larger range of stellar life spans. Uniform sampling in time from the truncated distributions thus results in a distribution of $F_{\mathrm{H} \alpha} / F_{\mathrm{FUV}}$ that is strongly peaked at the plateau value. Extinction adjusts the model values of $F_{\mathrm{H} \alpha} / F_{\mathrm{FUV}}$ slightly higher but is not strong enough in the outermost bin to create a noticeable high $F_{\mathrm{H} \alpha} / F_{\mathrm{FUV}}$ tail.

In summary, we find that while IMFs truncated as low as $50 M_{\odot}$ can qualitatively reproduce the observed distributions of $F_{\mathrm{H} \alpha} / F_{\mathrm{FUV}}$ in $\mathrm{HII}$ regions throughout $\mathrm{M} 101$, they produce no better agreement than a standard Kroupa IMF.

\section{Discussion}

We have shown that the distribution of the $F_{\mathrm{H} \alpha} / F_{\mathrm{FUV}}$ ratio in $\mathrm{H}$ II region populations, aside from extinction effects, does not change with environment in the M101 Group. We have also shown that we can model the observed distributions of $F_{\mathrm{H} \alpha} / F_{\mathrm{FUV}}$ in $\mathrm{H}$ II regions throughout the M101 Group without invoking a truncated IMF. The makeup of stellar populations ionizing H II regions throughout the M101 Group therefore appears ignorant of the local surface mass density; only the intensity of star formation changes.

We thus consider the possible origin of trends in $F_{\mathrm{H} \alpha} / F_{\mathrm{FUV}}$ with, e.g., galaxy stellar mass and central surface brightness found by other authors (e.g., Lee et al. 2009; Meurer et al. 2009). Because these studies focus on the integrated $\mathrm{H} \alpha$ and FUV fluxes of galaxies-which includes compact H II regions, DIG, and diffuse FUV emission-we consider how the DIG, diffuse FUV emission, and bias in measurement 
techniques might each contribute to the observed trends in integrated $F_{\mathrm{H} \alpha} / F_{\mathrm{FUV}}$ of whole galaxies. Finally, we discuss our results in the context of the M101 Group itself, particularly its tidal interaction history, and consider whether or not our results for this group can be generalized to other systems.

\subsection{On the Observed Trends of Integrated $F_{H \alpha} / F_{F U V}$}

If the IMF does not change with environment, as we have argued, why then do many studies find that $F_{\mathrm{H} \alpha} / F_{\mathrm{FUV}}$ integrated over galaxies or azimuthally averaged in wide radial bins is lower in low-density environments (e.g., Gil de Paz et al. 2005; Thilker et al. 2005; Lee et al. 2009; Meurer et al. 2009; Goddard et al. 2010)? Because the integrated $F_{\mathrm{H} \alpha} / F_{\mathrm{FUV}}$ includes all sources of $\mathrm{H} \alpha$ and FUV emission-from $\mathrm{H}$ II regions, to DIG, to diffuse FUV with no $\mathrm{H} \alpha$ counterpart-changes in integrated $F_{\mathrm{H} \alpha} / F_{\mathrm{FUV}}$ can result from many different factors, from variations in the IMF, to stochastic sampling of the IMF in low-mass clusters, to a nonuniform SFH (e.g., Lee et al. 2009; PflammAltenburg et al. 2009; Alberts et al. 2011; Barnes et al. 2011, 2013; Weisz et al. 2012; da Silva et al. 2014).

We have shown that variation in the IMF is unlikely within the M101 Group. Given our low resolution, most (>90\%) of the $\mathrm{H}$ II region complexes we identified have fluxes above where stochastic sampling ought to be important (e.g., Hermanowicz et al. 2013). However, a nonuniform SFH could result in abundant populations of FUV-emitting stars with no $\mathrm{H} \alpha$ counterpart, specifically if such populations are remnants of a fading burst of star formation. Therefore, given that $F_{\mathrm{H} \alpha} / F_{\mathrm{FUV}}$ is roughly constant in $\mathrm{H}$ II regions, we test for an overabundance of FUV relative to $\mathrm{H} \alpha$ by comparing the fractions of diffuse $\mathrm{H} \alpha$ emission (or the DIG fraction, hereafter $f_{\text {DIG }}$ ) and diffuse FUV emission (hereafter $f_{\text {DUV }}$ ). We define "diffuse" emission as any H $\alpha$ or FUV emission located outside of what we have defined as $\mathrm{H}$ II regions, for simplicity; hence, we measure $f_{\text {DIG }}$ and $f_{\text {DUV }}$ by masking out $\mathrm{H}$ II regions.

For the purposes of this study, we are concerned mainly with the relative values of the diffuse fractions between environments; hence, it is important only that we measure $f_{\text {DIG }}$ and $f_{\text {DUV }}$ in a consistent manner for each environment. However, given that we base our mask on the low-resolution GALEX imaging, it is useful to compare our value of $f_{\text {DIG }}$ with that found in other studies to estimate how much of the DIG directly adjacent to $\mathrm{H}$ II regions we could be masking. Given our canonical mask (4!. 5 apertures), we find a DIG fraction of $33 \%$. Our masking thus appears to be more aggressive than that of previous studies of the DIG (e.g., Thilker et al. 2002, who found a DIG fraction of $43 \%$ in M101), implying that with our canonical mask we are isolating the most diffuse part of the DIG. Changing the mask aperture by \pm 1 !' 5 yields changes in $f_{\text {DIG }}$ and $f_{\text {DUV }}$ of $\pm 20 \%$.

To test the influence of this masking on the relative fractions of DIG and diffuse FUV, we measured the azimuthally averaged radial profile of $F_{\mathrm{H} \alpha} / F_{\mathrm{FUV}}$ in the DIG in both M101 and NGC 5474. We found that both galaxies display a distinct downward trend in $F_{\mathrm{H} \alpha} / F_{\mathrm{FUV}}$ with radius-implying dominant diffuse FUV emission in their outer disks - that persists even when using an unrealistically aggressive mask that results in $f_{\text {DIG }} \sim 6 \%$ for M101. Therefore, our masking procedure does not appear to influence the results we present here. We defer a more detailed discussion of the DIG in the M101 Group to a forthcoming paper.
Table 2

Integrated Properties of M101 Group Galaxies

\begin{tabular}{lrrrrr}
\hline \hline Region & M101 & M101 & M101 & NGC 5474 & NGC 5477 \\
\hline$f_{\text {DIG }}$ & 0.33 & 0.34 & 0.28 & 0.17 & 0.20 \\
$f_{\text {DUV }}$ & 0.56 & 0.51 & 0.60 & 0.42 & 0.45 \\
$F_{\mathrm{H} \alpha} / F_{\mathrm{FUV}}$ & -1.98 & -2.08 & -1.95 & -2.29 & -2.14 \\
$F_{\mathrm{H} \alpha} / F_{\mathrm{FUV}}$, & -2.21 & -2.22 & -2.21 & -2.48 & -2.22 \\
$\quad$ & & & & & \\
\hline
\end{tabular}

Note. First row: fraction of $\mathrm{H} \alpha$ flux from the DIG. Second row: fraction of diffuse FUV emission. Third row: integrated $F_{\mathrm{H} \alpha} / F_{\mathrm{FUV}}$. Fourth row: integrated $F_{\mathrm{H} \alpha} / F_{\mathrm{FUV}}$ corrected for extinction. Systematic uncertainties, which dominate, are discussed in the text.

While the expectation is that high $f_{\text {DUV }}$ relative to $f_{\text {DIG }}$ should yield lower integrated $F_{\mathrm{H} \alpha} / F_{\mathrm{FUV}}$ ratios, we find that this is not always the case, implying that there may be methodological bias at play as well in measuring integrated properties of galaxies and regions of galaxies. Specifically, a bias may be incurred when using flux-weighted values over, e.g., areal-weighted values of $\mathrm{H} \alpha$ and FUV flux.

In Table 2, we give diffuse fractions in five environments in the M101 Group: M101 as a whole, its inner disk, its outer disk, its more distant companion NGC 5474, and its nearby dIrr companion NGC 5477. We measure both $f_{\text {DIG }}$ and $f_{\text {DUV }}$ in an identical manner; hence, they are comparable regardless of uncertainty in, e.g., the choice of $\mathrm{H}$ II region mask. Additionally, for each region, we give integrated values of $F_{\mathrm{H} \alpha} / F_{\mathrm{FUV}}$ before and after applying an extinction correction. In this case, we apply an integrated correction measured using the integrated FUV-NUV colors of each region, as is typically done in galaxy survey studies (e.g., Lee et al. 2009; Meurer et al. 2009).

Comparison of the diffuse fractions in $\mathrm{H} \alpha$ and $\mathrm{FUV}$ indicates that diffuse FUV emission is more prevalent compared to DIG in M101's outer disk and in both companion galaxies. This concurs with a visual examination of the images; in M101's outer disk, for example, we find many large (several kpc wide) patches of diffuse FUV emission that have no $\mathrm{H} \alpha$ counterpart in our difference image. The areal covering fraction of diffuse FUV emission appears larger than the DIG covering fraction across the whole outer disk, while in the inner disk, the covering fractions of both are roughly equal. Quantitatively, this is observable as a larger outer disk scale length in the FUV compared to the $\mathrm{H} \alpha$, such as is typically seen in other XUV disks (Gil de Paz et al. 2005; Thilker et al. 2005; Goddard et al. 2010).

However, the integrated values of $F_{\mathrm{H} \alpha} / F_{\mathrm{FUV}}$ do not reflect this. Despite the larger fractions of older FUV-emitting populations in M101's outer disk and the two companion galaxies, after correcting for extinction, only NGC 5474 shows a significantly different value of integrated $F_{\mathrm{H} \alpha} / F_{\mathrm{FUV}}$. This appears to be an artifact of the flux-weighted measurement; in NGC 5474, we find that the brightest $10 \%$ of H II regions (only 16 regions) contribute nearly $60 \%$ of the galaxy's total $\mathrm{H} \alpha$ flux. Thus, if something is systematically different about these few regions-age, dust content-compared to the remaining $\mathrm{H}$ II regions in the galaxy, this difference will drive the galaxy's flux-weighted mean $F_{\mathrm{H} \alpha} / F_{\text {FUV }}$ ratio to an unrepresentative value. In NGC 5474, the brightest $\mathrm{H}$ II regions have redder FUV-NUV colors $(\sim 0.05$ compared to $\sim-0.1$ in the dimmer 
regions). Because we derive the extinction based on the UV color, these regions are measured as dustier environments; if so, the extinction correction may be overcompensating for dust throughout NGC 5474 and driving the integrated $F_{\mathrm{H} \alpha} / F_{\mathrm{FUV}}$ down.

This is demonstrated in an alternative way in M101's outer disk. As in NGC 5474, the brightest regions in M101's outer disk are redder in UV color $(\sim-0.05$ compared to $\sim-0.2)$, hence potentially dustier, and again contribute a large fraction of the region's total $\mathrm{H} \alpha$ flux (40\%). Before applying an extinction correction, the median $F_{\mathrm{H} \alpha} / F_{\mathrm{FUV}}$ value of all of the H II regions in M101's outer disk is -2.32 , but the fluxweighted mean value of the $\mathrm{H}$ II regions is -2.09 . Fluxweighting thus drives the integrated $F_{\mathrm{H} \alpha} / F_{\mathrm{FUV}}$ ratio of $\mathrm{H}$ II regions in M101's outer disk to a higher value, as it is biased by the brighter, redder regions, in direct analogy with the integrated $F_{\mathrm{H} \alpha} / F_{\mathrm{FUV}}$ value of NGC 5474 .

As such, it is unclear whether or not the trends in integrated $F_{\mathrm{H} \alpha} / F_{\mathrm{FUV}}$ with stellar mass, SFR, and surface brightness noted in other studies result from physical changes or purely from systematics induced by the flux-weighted measurements. Regardless, the M101 Group is a well-studied system, with constraints on stellar populations throughout its disk and on its tidal interaction history with its companions (e.g., Beale \& Davies 1969; Rownd et al. 1994; Waller et al. 1997; Mihos et al. 2013). We can therefore make more specific conclusions about how M101's local environment may have influenced the star formation taking place in its outer disk and companions and consider whether or not these conclusions can be generalized to other similar systems. We discuss this further in the following section.

\subsection{The M101 Group as a Case Study}

We have shown that in the M101 Group, H II regions have roughly constant $F_{\mathrm{H} \alpha} / F_{\mathrm{FUV}}$ distributions regardless of their environment. We have also shown that diffuse FUV emission, with no $\mathrm{H} \alpha$ counterpart, is abundant in M101's outer disk and two companions, implying widespread populations of slightly older $\mathrm{O}$ and $\mathrm{B}$ stars in the field, similar to other XUV disks (e.g., Gil de Paz et al. 2005; Thilker et al. 2005). We argue here that this can be explained in the context of M101's interaction history and consider whether or not star formation in the lowdensity environments of the M101 Group could be representative of low-density environments as a whole.

In general, the origin of field $\mathrm{O}$ and $\mathrm{B}$ stars is not yet clear. They may form in situ (de Wit et al. 2005; Lamb et al. 2010; Oey et al. 2013) or within H II regions but be ejected at high velocity (Gies 1987; Moffat et al. 1998; de Wit et al. 2005), or they may be young clusters that have fully succeeded in clearing out gas and dust from their birth $\mathrm{H}$ II regions. In a study of diffuse FUV emission in the interarm regions of M101's inner disk, Crocker et al. (2015) found that the majority is likely emitted by $10-50$ Myr old stellar populations that have drifted from their birthplaces in spiral arms. Because these stars are carried by the disk's underlying rotation, the difference between the rotation speed and the spiral arm pattern speed determines how far they might travel from a given spiral arm; one might expect stars to remain very close to spiral arms near corotation, for instance.

UV light scattered into our line of sight by dust contributes a sizable fraction of the diffuse UV as well (upward of $~ 60 \%$; Crocker et al. 2015), but only in the vicinity of spiral arms; in a field adjacent to a spiral arm, Crocker et al. (2015) estimated that the UV flux contributed by scattered light drops by a factor of roughly 1.5 over a distance of $\sim 1.5 \mathrm{kpc}$. This, along with lower dust content, implies that the diffuse FUV in M101's outer disk contains very little scattered light. For example, in the galaxy's northeast, we find large patches (several kpc on a side) of diffuse FUV located some 5-10 kpc from the nearest spiral arm, as well as some a similar distance from the nearest H II region. This FUV emission thus appears to be a remnant of a previous episode of star formation, which either formed in situ or migrated from elsewhere in the disk. The largest such patch $(\sim 2 \mathrm{kpc}$ in radius, detected at $>10 \sigma$ significance in the FUV) has an FUV-NUV color of $\sim 0.6$; in a model of color evolution in integrated populations by Boissier et al. (2008), young populations maintain an FUV-NUV color of $\sim 0.0$ while SF is ongoing and reach $\sim 0.6$ roughly $200 \mathrm{Myr}$ after star formation begins to decline (neglecting extinction, although extinction may be safely neglected in outer disks). In the Milky Way, populations of $\mathrm{O}$ and $\mathrm{B}$ stars have radial velocity dispersions of order $\sim 10 \mathrm{~km} \mathrm{~s}^{-1}$ (Binney \& Merrifield 1998) and thus can easily disperse over $\sim 2 \mathrm{kpc}$ in radius in $200 \mathrm{Myr}$. This diffuse FUV-emitting starlight thus likely formed in a localized burst a few hundred Myr ago and is now beginning to fade. We find many other such patches of diffuse FUV throughout M101's outer disk with similarly red colors $(\sim 0.4-0.6)$, implying similar origins.

M101's disturbed morphology implies that it suffered a recent tidal interaction. From the integrated $B-V$ colors in its outer disk, Mihos et al. (2013) proposed that this morphology resulted from a flyby encounter with its more distant companion NGC 5474 some $\sim 300 \mathrm{Myr}$ ago, resulting in a brief and currently fading burst of star formation. After $300 \mathrm{Myr}$, even the NUV light begins to fade; $\mathrm{H} \alpha$ emission would thus be scarce, as it is in the diffuse FUV patches discussed above. Follow-up HST imaging of stellar populations in M101's northeast plume region are consistent with this star formation timeline (J. C. Mihos et al. 2017, in preparation), providing strong support that star formation in M101's outer disk was induced by an interaction. This in turn shows that M101's outer disk does not have a uniform SFH. If NGC 5474 was the culprit in the interaction, it too should have seen a starburst on the same timescale, hence it too should have a nonuniform SFH. The M101 Group hence provides a fairly clear example of an FUV-dominated outer disk resulting from a fading, tidally induced starburst; from this perspective, too, it is not necessary to invoke changes in the IMF to explain the star-forming properties of the M101 Group.

Is this scenario generalizable to other systems? XUV disks are often suggested to be tidal in origin (Gil de Paz et al. 2005; Thilker et al. 2005, 2007) or created through gas accretion into the outer disk (Lemonias et al. 2011). Also, the UV emission in XUV disks is typically concentrated in filments reminiscent of spiral structure (Thilker et al. 2007). While outer disks may typically be stable against spiral arm formation, we can consider the longevity of a set of spiral arms induced in an outer disk by a tidal interaction, hence the longevity of XUV disks in general. As a rough estimate, let us assume that spiral arms in outer disks are not self-sustaining due to high disk stability (e.g., Kennicutt 1989) and so lose their coherency over one dynamical time; in M101 at $16 \mathrm{kpc}$ (roughly where we demarcate its outer disk), this is $\sim 500 \mathrm{Myr}$ (assuming a rotation speed of $\sim 190 \mathrm{~km} \mathrm{~s}^{-1}$; Meidt et al. 2009). Star 
formation persists in M101 out to $\sim 40 \mathrm{kpc}$, where a dynamical time is $\sim 1.3 \mathrm{Gyr}$. As such, if galaxies like M101 suffer only one interaction in their lifetimes capable of producing an XUV disk, assuming a total lifetime of $\sim 10 \mathrm{Gyr}$, there would be an $\sim 5 \%-13 \%$ probability that we would witness it in this state at $z=0$. A study by Lemonias et al. (2011) found that XUV disks exist in $4 \%-14 \%$ of galaxies out to $z=0.05$; if M101 can be considered representative (it is slightly brighter than $L^{*}$ in the $V$ band; de Vaucouleurs et al. 1991), then the average galaxy need suffer only one to two interactions capable of producing XUV disks in its lifetime to explain the frequency of XUV disks in the local universe. Whether or not this is reasonable depends on how specific the parameters of the interactions must be in order to produce an XUV disk (mass ratio, relative inclination, relative velocities, etc.), but this simple argument suggests that all XUV disks may be explainable through tidal interactions with satellites.

Even in purely isolated systems, the global stability of outer disks means that some manner of perturbation is still necessary to initiate star formation there (e.g., substructure in the dark matter halo; Bush et al. 2010). If star formation in dwarf galaxies results mainly from, e.g., supernova feedback (van Zee et al. 1997), some manner of perturbation would be required to initiate it in the first place. This general dependence on external forces, rather than on potentially long-lived, regularly rotating spiral features or bars, implies that star formation in lowdensity environments may always be subject to stochasticity; hence, an assumption of constant star formation over Gyr timescales in such environments could be highly suspect. As more and more systems are studied, and as finer and finer resolution SFHs are obtained of these systems, the nature of star formation and the evolution of galaxies should begin to become clear.

\section{Summary}

We have performed a study of star formation across all environments in the nearby M101 Group-M101's inner disk, outer disk, and two lower-mass companions-using both new deep $\mathrm{H} \alpha$ narrowband imaging and archival UV data (GALEX NGS and PI data; Bianchi et al. 2003; Bigiel et al. 2010) in order to test whether or not star formation physics (specifically the IMF) changes with environment. We have chosen to study only the $\mathrm{H} \alpha$-emitting $\mathrm{H}$ II regions in these environments in order to compare only populations young enough to retain their most massive stars.

We have performed photometry on all $\mathrm{H}$ II regions in M101, NGC 5474, and NGC 5477 in order to measure their $F_{\mathrm{H} \alpha} / F_{\mathrm{FUV}}$ ratios, which should be systematically low in the absence of massive $\left(M \sim 20-100 M_{\odot}\right)$ stars. We find that the median $F_{\mathrm{H} \alpha} / F_{\mathrm{FUV}}$ ratio across all populations of $\mathrm{H}$ II regions in the M101 Group does not vary significantly, once bulk radial extinction trends are taken into account. In addition to the median, however, we also find that the scatter in $F_{\mathrm{H} \alpha} / F_{\mathrm{FUV}}$ does not vary significantly with environment. While typical H II region fluxes do decline with radius in M101 and its larger companion NGC 5474, their near-constant distribution of $F_{\mathrm{H} \alpha} / F_{\mathrm{FUV}}$ ratios implies that the populations of ionizing stars-even in the fainter outer disk H II regions-are being sampled from the same IMF as in the inner disk. The decline in mean $\mathrm{H}$ II region flux may thus be attributable primarily to a decline in mean surface gas density alone, rather than any significant change in the cloud-to-cloud physics of star formation.

Using Starburst99 models (Leitherer et al. 1999), we attempt to determine whether or not a truncated IMF was necessary to reproduce the observed distributions of $\mathrm{H}$ II regions in any radial bin in $\mathrm{M} 101$. We find that, while we are able to qualitatively reproduce these distributions using IMFs truncated at the high-mass end $\left(\leqslant 50 M_{\odot}\right)$, we are able to just as successfully reproduce the distributions of $F_{\mathrm{H} \alpha} / F_{\mathrm{FUV}}$ using a standard Kroupa IMF, regardless of the local surface brightness. It therefore appears that, at least when comparing bulk populations, it is unnecessary to invoke changes to the IMF to explain the properties of the H II regions in M101.

Assuming that the IMF is universal, we further investigate the origin of trends in integrated $F_{\mathrm{H} \alpha} / F_{\mathrm{FUV}}$ with surface brightness, SFR, and stellar mass found by other authors (e.g., Thilker et al. 2005; Boselli et al. 2009; Lee et al. 2009; Meurer et al. 2009; Goddard et al. 2010). Because the $F_{\mathrm{H} \alpha} / F_{\mathrm{FUV}}$ ratios of $\mathrm{H}$ II regions do not change throughout the M101 Group, we compare the relative fractions of diffuse $\mathrm{H} \alpha$ and FUV emission and find that, indeed, diffuse FUV emission with no $\mathrm{H} \alpha$ counterpart-and hence no extremely young, massive stars-is more prevalent in lower surface density regions. This implies that such regions suffered a recent but now fading burst of star formation and hence are not necessarily continuously forming new stars; low integrated $F_{\mathrm{H} \alpha} / F_{\mathrm{FUV}}$ ratios in these low-density regions may thus result from a bursty or otherwise nonuniform SFH. However, we have also shown that using flux-weighted $F_{\mathrm{H} \alpha} / F_{\mathrm{FUV}}$ or extinction corrections can bias the value of the integrated $F_{\mathrm{H} \alpha} / F_{\mathrm{FUV}}$ in galaxies, particularly if the bulk of the $\mathrm{H} \alpha$ or FUV intensity emerges from a small number of bright $\mathrm{H}$ II regions. We thus advise caution in future such studies with regard to how integrated $F_{\mathrm{H} \alpha} / F_{\mathrm{FUV}}$ is measured and corrected for extinction.

Finally, we consider whether or not the M101 Group could be exceptional or whether our results are more broadly applicable. Previous studies have shown that star formation in M101's outer disk was likely triggered by a tidal interaction several hundred Myr ago (Mihos et al. 2013). This lends credence to the interpretation that the abundant populations of FUV-emitting stars with no $\mathrm{H} \alpha$ counterpart are remnants of a now-fading burst of star formation. Additionally, we have shown that if interaction-induced star formation in outer disks persists over only one dynamical time, it may still be long-lived enough to account for the low frequency (4\%-14\%; Lemonias et al. 2011) of XUV disks observed in the local universe. Therefore, assuming that the conditions necessary to create XUV disks through tidal interactions are not oddly specific, it is not unreasonable to consider that all XUV disks may be tidally induced. If so, this would imply that star formation in lowdensity environments only differs from star formation in highdensity environments in that it requires outside perturbation to be initiated. This implies in turn that star formation at low density is subject to greater stochasticity than star formation at high density; assuming a uniform SFH in the low-density regime may be unwise.

This work has been supported by the National Science Foundation through award 1108964 to JCM, as well as through award 1211144 to PH. This work made use of Numpy, Scipy (Oliphant 2007), MatPlotLib (Hunter 2007), astroML (VanderPlas et al. 2012), and the community-developed core 
Python package for astronomy, Astropy (Astropy Collaboration et al. 2013). We would like to thank Heather Morrison for many helpful discussions regarding the statistical analyses presented in this work, as well as Stacy McGaugh, Sally Oey, and Daniella Calzetti for helpful discussions about extinction, star formation physics, and star formation tracers. We would also like to thank the anonymous referee for a very thorough and helpful review, which we feel greatly improved this manuscript.

Facilities: CWRU:Schmidt-The Burrell Schmidt of the Warner and Swasey Observatory, Case Western Reserve University; GALEX.

\section{ORCID iDs}

\section{J. Christopher Mihos (i) https://orcid.org/0000-0002- 7089-8616}

Paul Harding (i) https://orcid.org/0000-0003-3442-6248

\section{References}

Aihara, H., Allende Prieto, C., An, D., et al. 2011, ApJS, 193, 29 Alberts, S., Calzetti, D., Dong, H., et al. 2011, ApJ, 731, 28

Astropy Collaboration, Robitaille, T. P., Tollerud, E. J., et al. 2013, A\&A, 558, A33

Bakos, J., Trujillo, I., \& Pohlen, M. 2008, ApJL, 683, L103

Barker, M. K., Sarajedini, A., Geisler, D., Harding, P., \& Schommer, R. 2007, AJ, 133, 1125

Barnes, K. L., van Zee, L., \& Dowell, J. D. 2013, ApJ, 775, 40

Barnes, K. L., van Zee, L., \& Skillman, E. D. 2011, ApJ, 743, 137

Beale, J. S., \& Davies, R. D. 1969, Natur, 221, 531

Bertin, E., \& Arnouts, S. 1996, A\&AS, 117, 393

Bianchi, L., Madore, B., Thilker, D., Gil de Paz, A. \& GALEX Science Team 2003, BAAS, 35, 1354

Bigiel, F., Leroy, A., Walter, F., et al. 2008, AJ, 136, 2846

Bigiel, F., Leroy, A., Walter, F., et al. 2010, AJ, 140, 1194

Binney, J., \& Merrifield, M. 1998, Galactic Astronomy (Princeton, NJ: Princeton Univ. Press)

Boissier, S., Gil de Paz, A., Boselli, A., et al. 2008, ApJ, 681, 244

Bolatto, A. D., Leroy, A. K., Jameson, K., et al. 2011, ApJ, 741, 12

Bonnell, I. A., Vine, S. G., \& Bate, M. R. 2004, MNRAS, 349, 735

Boselli, A., Boissier, S., Cortese, L., et al. 2009, ApJ, 706, 1527

Bottema, R., Shostak, G. S., \& van der Kruit, P. C. 1987, Natur, 328, 401

Breiman, L. 1973, Statistics with a View toward Applications (Boston: Houghton Mifflin)

Bressan, A., Marigo, P., Girardi, L., et al. 2012, MNRAS, 427, 127

Brown, A. G. A., de Geus, E. J., \& de Zeeuw, P. T. 1994, A\&A, 289, 101

Brown, B. W., \& Forsythe, A. B. 1974, J. Am. Stat. Assoc., 69, 364

Bruzzese, S. M., Meurer, G. R., Lagos, C. D. P., et al. 2015, MNRAS, 447, 618

Burkholder, V., Impey, C., \& Sprayberry, D. 2001, AJ, 122, 2318

Bush, S. J., Cox, T. J., Hayward, C. C., et al. 2010, ApJ, 713, 780

Calzetti, D. 2001, PASP, 113, 1449

Calzetti, D., Kennicutt, R. C., Jr., Bianchi, L., et al. 2005, ApJ, 633, 871

Cortese, L., Boselli, A., Buat, V., et al. 2006, ApJ, 637, 242

Courtes, G., \& Cruvellier, P. 1961, POHP, 5, 42

Crocker, A. F., Chandar, R., Calzetti, D., et al. 2015, ApJ, 808, 76

Croxall, K. V., Pogge, R. W., Berg, D. A., Skillman, E. D., \& Moustakas, J. 2016, ApJ, 830, 4

da Silva, R. L., Fumagalli, M., \& Krumholz, M. R. 2014, MNRAS, 444, 3275

Davidge, T. J. 2003, AJ, 125, 3046

Davidge, T. J. 2010, ApJ, 718, 1428

de Vaucouleurs, G., de Vaucouleurs, A., Corwin, H. G., Jr., et al. 1991, Third Reference Catalogue of Bright Galaxies (New York: Springer)

de Wit, W. J., Testi, L., Palla, F., \& Zinnecker, H. 2005, A\&A, 437, 247

Debattista, V. P., Mayer, L., Carollo, C. M., et al. 2006, ApJ, 645, 209

Donas, J., Deharveng, J. M., Laget, M., Milliard, B., \& Huguenin, D. 1987, A\&A, 180, 12

Dopita, M. A., \& Sutherland, R. S. 2003, Astrophysics of the Diffuse Universe (Berlin: Springer)

Dyson, J. E., \& Williams, D. A. 1980, Physics of the Interstellar Medium (2nd ed.; New York: Halsted Press)

Elmegreen, B. G., \& Hunter, D. A. 2015, ApJ, 805, 145

Ferguson, A. M. N., Gallagher, J. S., \& Wyse, R. F. G. 1998, AJ, 116, 673
Finlator, K., Ivezić, Ž, Fan, X., et al. 2000, AJ, 120, 2615

García-Ruiz, I., Sancisi, R., \& Kuijken, K. 2002, A\&A, 394, 769

Garcia-Segura, G., \& Franco, J. 1996, ApJ, 469, 171

Gies, D. R. 1987, ApJS, 64, 545

Gil de Paz, A., Madore, B. F., Boissier, S., et al. 2005, ApJL, 627, L29

Goddard, Q. E., Kennicutt, R. C., \& Ryan-Weber, E. V. 2010, MNRAS, 405, 2791

Gunawardhana, M. L. P., Hopkins, A. M., Sharp, R. G., et al. 2011, MNRAS, 415,1647

Haffner, L. M., Dettmar, R.-J., Beckman, J. E., et al. 2009, RvMP, 81, 969

Heckman, T., Krolik, J., Meurer, G., et al. 1995, ApJ, 452, 549

Helmboldt, J. F., Walterbos, R. A. M., Bothun, G. D., \& O’Neil, K. 2005, ApJ, 630,824

Helmboldt, J. F., Walterbos, R. A. M., Bothun, G. D., O’Neil, K., \& Oey, M. S. 2009, MNRAS, 393, 478

Hermanowicz, M. T., Kennicutt, R. C., \& Eldridge, J. J. 2013, MNRAS, 432, 3097

Hoopes, C. G., Walterbos, R. A. M., \& Bothun, G. D. 2001, ApJ, 559, 878

Hoversten, E. A., \& Glazebrook, K. 2008, ApJ, 675, 163

Huber, P. J. 1981, Robust Statistics (New York: Wiley)

Hunter, D. A., Elmegreen, B. G., Dupuy, T. J., \& Mortonson, M. 2003, AJ, 126,1836

Hunter, D. A., Elmegreen, B. G., \& Ludka, B. C. 2010, AJ, 139, 447

Hunter, D. A., Elmegreen, B. G., Oh, S.-H., et al. 2011, AJ, 142, 121

Hunter, D. A., \& Gallagher, J. S., III 1986, PASP, 98, 5

Hunter, D. A., \& Plummer, J. D. 1996, ApJ, 462, 732

Hunter, J. D. 2007, CSE, 9, 90

Kellar, J. A., Salzer, J. J., Wegner, G., Gronwall, C., \& Williams, A. 2012, AJ, 143,145

Kenney, J. D. P., Scoville, N. Z., \& Wilson, C. D. 1991, ApJ, 366, 432

Kennicutt, R. C., \& Evans, N. J. 2012, ARA\&A, 50, 531

Kennicutt, R. C., Jr. 1983, ApJ, 272, 54

Kennicutt, R. C., Jr. 1989, ApJ, 344, 685

Kennicutt, R. C., Jr. 1998, ApJ, 498, 541

Kennicutt, R. C., Jr., Calzetti, D., Walter, F., et al. 2007, ApJ, 671, 333

Kennicutt, R. C., Jr., Lee, J. C., Funes, J. G., et al. 2008, ApJS, 178, 247

Kennicutt, R. C., Jr., Tamblyn, P., \& Congdon, C. E. 1994, ApJ, 435, 22

Kornreich, D. A., Haynes, M. P., \& Lovelace, R. V. E. 1998, AJ, 116, 2154

Koyama, Y., Kodama, T., Hayashi, M., et al. 2015, MNRAS, 453, 879

Kroupa, P. 2001, MNRAS, 322, 231

Lada, C. J., \& Lada, E. A. 2003, ARA\&A, 41, 57

Laine, J., Laurikainen, E., \& Salo, H. 2016, A\&A, 596, A25

Lamb, J. B., Oey, M. S., Werk, J. K., \& Ingleby, L. D. 2010, ApJ, 725, 1886

Larson, R. B. 1973, MNRAS, 161, 133

Lee, J. C., Gil de Paz, A., Kennicutt, R. C., Jr., et al. 2011, ApJS, 192, 6

Lee, J. C., Gil de Paz, A., Tremonti, C., et al. 2009, ApJ, 706, 599

Leitherer, C., Schaerer, D., Goldader, J. D., et al. 1999, ApJS, 123, 3

Lemonias, J. J., Schiminovich, D., Thilker, D., et al. 2011, ApJ, 733, 74

Leroy, A. K., Bigiel, F., de Blok, W. J. G., et al. 2012, AJ, 144, 3

Levene, H. 1960, in Contributions to Probability and Statistics, ed. I. Olkin (Palo Alto, CA: Stanford Univ. Press), 278

Lim, T. S., \& Loh, W. Y. 1996, Comp. Stat. Data Analysis, 22, 287

Martin, C. L., \& Kennicutt, R. C., Jr. 2001, ApJ, 555, 301

Massey, P., \& Foltz, C. B. 2000, PASP, 112, 566

Massey, P., Strobel, K., Barnes, J. V., \& Anderson, E. 1988, ApJ, 328, 315

McGaugh, S. S., \& Bothun, G. D. 1994, AJ, 107, 530

McGaugh, S. S., \& de Blok, W. J. G. 1997, ApJ, 481, 689

Meidt, S. E., Rand, R. J., \& Merrifield, M. R. 2009, ApJ, 702, 277

Meurer, G. R., Heckman, T. M., \& Calzetti, D. 1999, ApJ, 521, 64

Meurer, G. R., Heckman, T. M., Leitherer, C., et al. 1995, AJ, 110, 2665

Meurer, G. R., Wong, O. I., Kim, J. H., et al. 2009, ApJ, 695, 765

Mihos, J. C., Harding, P., Feldmeier, J. J., et al. 2017, ApJ, 834, 16

Mihos, J. C., Harding, P., Spengler, C. E., Rudick, C. S., \& Feldmeier, J. J. 2013, ApJ, 762, 82

Mihos, J. C., Keating, K. M., Holley-Bockelmann, K., Pisano, D. J., \& Kassim, N. E. 2012, ApJ, 761, 186

Minchev, I., Famaey, B., Combes, F., et al. 2011, A\&A, 527, A147

Moffat, A. F. J., Marchenko, S. V., Seggewiss, W., et al. 1998, A\&A, 331, 949

Morrison, H. L., Flynn, C., \& Freeman, K. C. 1990, AJ, 100, 1191

Morrissey, P., Conrow, T., Barlow, T. A., et al. 2007, ApJS, 173, 682

Oey, M. S., Lamb, J. B., Kushner, C. T., Pellegrini, E. W., \& Graus, A. S. 2013, ApJ, 768, 66

Oliphant, T. E. 2007, CSE, 9, 10

Pflamm-Altenburg, J., González-Lópezlira, R. A., \& Kroupa, P. 2013, MNRAS, 435, 2604

Pflamm-Altenburg, J., \& Kroupa, P. 2008, Natur, 455, 641 
Pflamm-Altenburg, J., Weidner, C., \& Kroupa, P. 2009, MNRAS, 395, 394

Pilyugin, L. S., Grebel, E. K., \& Kniazev, A. Y. 2014, AJ, 147, 131

Quireza, C., Rood, R. T., Bania, T. M., Balser, D. S., \& Maciel, W. J. 2006, ApJ, 653, 1226

Reynolds, R. J. 1990, in Proc. IAU Symp. 139, The Galactic and Extragalactic Background Radiation, ed. S. Boyer \& C. Leinert (Melville, NY: IAU), 157

Roškar, R., Debattista, V. P., Quinn, T. R., Stinson, G. S., \& Wadsley, J. 2008, ApJL, 684, L79

Roškar, R., Debattista, V. P., Quinn, T. R., \& Wadsley, J. 2012, MNRAS, 426, 2089

Rownd, B. K., Dickey, J. M., \& Helou, G. 1994, AJ, 108, 1638

Rudick, C. S., Mihos, J. C., Harding, P., et al. 2010, ApJ, 720, 569

Sánchez-Blázquez, P., Courty, S., Gibson, B. K., \& Brook, C. B. 2009, MNRAS, 398, 591

Sancisi, R. 1976, A\&A, 53, 159

Schlafly, E. F., \& Finkbeiner, D. P. 2011, ApJ, 737, 103

Schlegel, D. J., Finkbeiner, D. P., \& Davis, M. 1998, ApJ, 500, 525

Schmidt, M. 1959, ApJ, 129, 243

Schombert, J., McGaugh, S., \& Maciel, T. 2013, AJ, 146, 41

Schönrich, R., \& Binney, J. 2009, MNRAS, 399, 1145

Schruba, A., Leroy, A. K., Walter, F., et al. 2011, AJ, 142, 37

Scowen, P. A., Dufour, R. J., \& Hester, J. J. 1992, AJ, 104, 92

Seibert, M., Martin, D. C., Heckman, T. M., et al. 2005, ApJL, 619, L55

Sellwood, J. A., \& Binney, J. J. 2002, MNRAS, 336, 785

Skillman, E. D. 1987, in NASA Conf. 2466, Star Formation in Galaxies, ed. C. J. Lonsdale Persson (Washington, DC: NASA), 263

Slater, C. T., Harding, P., \& Mihos, J. C. 2009, PASP, 121, 1267

Sullivan, M., Treyer, M. A., Ellis, R. S., \& Mobasher, B. 2004, MNRAS, 350,21

Thilker, D. A., Bianchi, L., Boissier, S., et al. 2005, ApJL, 619, L79
Thilker, D. A., Bianchi, L., Meurer, G., et al. 2007, ApJS, 173, 538

Thilker, D. A., Walterbos, R. A. M., Braun, R., \& Hoopes, C. G. 2002, AJ, 124,3118

Toomre, A. 1964, ApJ, 139, 1217

van der Hulst, J. M., \& Huchtmeier, W. K. 1979, A\&A, 78, 82

van der Hulst, J. M., Skillman, E. D., Kennicutt, R. C., \& Bothun, G. D. 1987, A\&A, 177, 63

van der Hulst, J. M., van Albada, T. S., \& Sancisi, R. 2001, in ASP Conf. Ser.

240, Gas and Galaxy Evolution, ed. J. E. Hibbard, M. Rupen, \&

J. H. van Gorkum (San Francisco, CA: ASP), 451

van der Kruit, P. C. 1987, A\&A, 173, 59

van Eymeren, J., Jütte, E., Jog, C. J., Stein, Y., \& Dettmar, R.-J. 2011, A\&A, 530, A30

van Zee, L., Haynes, M. P., Salzer, J. J., \& Broeils, A. H. 1997, AJ, 113, 1618 VanderPlas, J., Connolly, A. J., Ivezic, Z., \& Gray, A. 2012, in Proc. in Conf on Intelligent Data Understanding (CIDU), ed. K. Das, N. V. Chawla, \& A. N. Srivastava (Los Alamitos, CA: IEEE), 47

Vandervoort, P. O. 1970, ApJ, 161, 87

Vlajić, M., Bland-Hawthorn, J., \& Freeman, K. C. 2009, ApJ, 697, 361

Vlajić, M., Bland-Hawthorn, J., \& Freeman, K. C. 2011, ApJ, 732, 7

Vogel, S. N., Weymann, R., Rauch, M., \& Hamilton, T. 1995, ApJ, 441, 162

Waller, W. H., Bohlin, R. C., Cornett, R. H., et al. 1997, ApJ, 481, 169

Walter, F., Brinks, E., de Blok, W. J. G., et al. 2008, AJ, 136, 2563

Watkins, A. E., Mihos, J. C., \& Harding, P. 2016, ApJ, 826, 59

Watkins, A. E., Mihos, J. C., Harding, P., \& Feldmeier, J. J. 2014, ApJ, 791, 38

Weidner, C., Kroupa, P., \& Larsen, S. S. 2004, MNRAS, 350, 1503

Weisz, D. R., Johnson, B. D., Johnson, L. C., et al. 2012, ApJ, 744, 44

Whitmore, B. C., Chandar, R., Kim, H., et al. 2011, ApJ, 729, 78

Zaritsky, D., \& Christlein, D. 2007, AJ, 134, 135

Zasov, A. V., \& Simakov, S. G. 1988, Ap, 29, 518

Zheng, Z., Thilker, D. A., Heckman, T. M., et al. 2015, ApJ, 800, 120 